Bijdragen tot de Dierkunde, 58 (2): 205-226 - 1988

\title{
GENETIC DIFFERENTIATION OF THE IBERIAN AMPHIPODS GAMMARUS IBERICUS MARGALEF, 1951 AND G. GAUTHIERI S. KARAMAN, 1935, WITH REFERENCE TO SOME RELATED SPECIES IN FRANCE
}

\author{
by \\ MAARTEN SCHEEPMAKER, FRITS VAN DER MEER \& SJOUK PINKSTER \\ Institute of Taxonomic Zoology, University of Amsterdam, P.O. Box 4766, \\ 1009 AT Amsterdam, The Netherlands
}

\begin{abstract}
Populations of five related species belonging to the Gammarus pulex group from the Iberian Peninsula and southern France have been studied electrophoretically at 21 enzyme loci. Morphologically distinct forms from the same side of the Pyrenees proved to be genetically more similar than morphologically very similar forms from opposite sides of this barrier.

With reference to published data, genetic differentiation between French and Iberian species was observed at the generic level. Inter-areal intraspecific genetic differentiation in Iberian species may warrant recognition of subspecies or sibling species. However, cross-breeding experiments performed did not assess levels of reproductive isolation of the forms involved.

A dendrogram derived from genetic distances $(D)$ was compared with geological and paleoclimatological evidence. Although hypotheses derived from the alternative data bases do not conflict, they give little information with regard to evolutionary rates of the loci involved.
\end{abstract}

\section{RÉSUMÉ}

Des populations de cinq espèces apparentées appartenant au groupe Gammarus pulex provenant de la péninsule Ibérique et du Midi de la France ont été étudiées par électrophorèse à 21 loci d'enzymes. Des formes morphologiquement distinctes du même versant des Pyrénées se sont montrées génétiquement plus proches que des formes morphologiquement fort semblantes du versant opposé de cette barrière.

En se basant sur des données publiées, la différentiation génétique entre les espèces ibériques et françaises a été constatée au niveau générique. La différentiation génétique intra-spécifique entre aires de répartition dans le cas des espèces ibériques, peut permettre de reconnaître des sous-espèces ou des espèces-jumelles. Cependant, des essais d'hybridation effectuées n'ont pu établir une isolation reproductive, à divers niveaux, des formes concernées.

Un dendrogramme dérivé de distances génétiques $(D)$ a été confronté avec des données géologiques et paléoclima- tologiques. Bien que les hypothèses basées sur d'autres séries de données diverses ne se contredisent pas, elles apportent peu d'information sur les rapports évolutifs des loci concernés.

\section{INTRODUCTION}

The Gammarus pulex group (sensu Karaman \& Pinkster, 1977) of the Iberian Peninsula is represented by $G$. gauthieri S. Karaman, 1935 and G. ibericus Margalef, 1951. G. gauthieri is known from Tunisia, Algeria, Morocco and many more or less isolated localities in Spain (Pinkster, 1971). Until 1974 G. ibericus was known only from the type locality. In that year, the species was recorded from many localities in the Massif Central, France (Goedmakers, 1974). Indeed, individuals from these populations resembled $G$. ibericus in almost every morphological detail. Pinkster \& Scholl (1984), however, demonstrated that a sample of individuals from one of these localities (station 2 in the present study; table I, fig. 1A) was genetically distinct and reproductively isolated from $G$. ibericus. This form was described as $G$. orinos Pinkster \& Scholl, 1984.

Geologically, the Iberian Peninsula and the rest of the European continent have developed quite independently (e.g. Vandenberg, 1979; Dercourt et al., 1985). Moreover, it is well known that there exists a rather close relationship between the freshwater fauna of the Iberian Peninsula and that of North Africa (Margalef, 1983; Pinkster, 1971; García de Jalón Lastra \& González del Tánago, 1986). Reproductively isolated populations of the $G$. 
pulex group in France (Scheepmaker, unpublished) differ genetically much less from each other than $G$. orinos and $G$. ibericus (Pinkster \& Scholl, 1984). The possibility exists that morphologically rather different forms from the Iberian Peninsula (e.g. G. ibericus and G. gauthieri) may be genetically more similar than even morphologically more comparable forms on either (the Iberian and the European) side of the Pyrenees (i.e. G. ibericus versus $G$. orinos, G. stupendus, and a morphologically distinct form restricted to the Pyrenees which is referred to as $G$. cf. fossarum by Goedmakers \& Roux, 1975).

The primary aim of this study was to determine if the Iberian members of the $G$. pulex group form a genetic entity separate from those of the rest of the European continent. Secondly, as some members of the G. pulex group, although genetically homogeneous, show considerable morphological variation and vice versa (Scheepmaker, 1987), the possibility of geographically proximate populations of $G$. ibericus and $G$. gauthieri being closer related to each other than allopatric populations of $G$. gauthieri was investigated.

Genetic variation at 21 presumptive gene loci was investigated using starch gel enzyme electrophoresis. In order to evaluate the biological significance of the genetic variation recorded, cross-breeding experiments have been carried out between two geographically distanct populations of $G$. gauthieri, two genetically rather different populations of $G$. ibericus and two more or less sympatric populations of $G$. gauthieri and $G$. ibericus. From these data the relationships among the samples studied were evaluated.

\section{MATERIAL AND METHODS}

\section{Sampling and collection sites}

Sampling for electrophoretic studies and cross-breeding experiments was carried out based on the procedures of Scheepmaker (1987). The collection sites and sampling dates are listed in table I and fig. 1. Sampling localities for electrophoretic analysis have been chosen according to Pinkster (1971), Goedmakers (1974) and Goedmakers Roux (1975), who did extensive sampling on the Iberian Peninsula, in the Massif Central (central France) and in the northern Pyrenees (southern France). In the following, samples of individuals of populations will be referred to as "populations".

Morphologically similar forms of $G$. gauthieri were sampled in the Valladolid area (fig. 1C: stations 10, 11 and 12) and the Serranía de Cuenca (fig. 1D: stations 6 and 7). Morphologically, populations from these areas differ slightly in the setation of P5-P7 (figs. 2D, E). Except for the length of the inner ramus of uropod 3 (fig. $2 \mathrm{~N}$ ), populations from both areas resemble $G$. fossarum Koch in many aspects.

G. ibericus differs from $G$. gauthieri by the setosity of P5P7 (figs. 2D, E, F) and the absence of setae on the dorsal side of the telson (figs. $2 \mathrm{Q}, \mathrm{S}$ ). Morphologically identical populations of $G$. ibericus were sampled in different drainage systems in the Serranía de Cuenca (table I, fig. 1D: stations 8 and 9). G. orinos (table I, fig. 1A: station 2), $G$. cf. fossarum (table I, fig. 1B: stations $3,4,5$ ) and $G$. stupendus (table I, fig. 1A: station 1) are morphologically related forms of which above all $G$. orinos resembles $G$. ibericus in many details (e.g. figs. 2G, F, M, O). Comparison of the differentiation among these forms as for morphological characters is summarized in table II.

\section{Electrophoresis}

The genetic variation at 21 enzyme loci was studied using both horizontal and vertical starch gel electrophoresis. Electrophoresis generally followed Siegismund et al., 1985 (horizontal starch gel electrophoresis) and Bulnheim \& Scholl, 1981 (vertical starch gel electrophoresis). Starch gels (Connaught $12 \% \mathrm{w} / \mathrm{v}$ ) were run for $15-17$ hours at 20 $\mathrm{V} \mathrm{cm}-1$ (buffer IV; $8 \mathrm{~V} \mathrm{~cm}^{-1}$ for vertical electrophoresis), 5 to $6 \mathrm{~V} \mathrm{~cm}^{-1}$ (buffers I, II, III, V and VI) and 3 to $4 \mathrm{~V}$ $\mathrm{cm}^{-1}$ (buffer VII). The enzymes assayed are enumerated in table III along with the corresponding buffer systems. Staining techniques of ADA, ALP, APK, EST, GOT, GPI, LAP, MDH, ME, MPI, and PEP in general followed Siegismund et al. (1985). The segregation of the Est-1 and Est-2 loci requires long pathways. Therefore, in horizontal electrophoresis, extra long molds were used. Samples from station 1 serve as "marker" in all our studies. Thus, sample sizes of station 1 are nearly always exceeding 99 (table IV).

Individuals in some samples gave very poor results for the $\mathrm{GDH}, \mathrm{MDH}$ and $\mathrm{ME}$ stainings, thus the substrate concentration was generally doubled. For the PEP stainings, 1-leucyl-glycyl-glycine and 1-leucyl-glycyl-tyrosine were used as substrate. GDH, HK, 6PGD and PK stainings were according to Shaw \& Prasad (1970), Jelnes (1971), Giblett (1969) and modified after Brewer (1970), respectively.

\section{Analysis of allozyme variation}

Genetic interpretation was inferential. Electromorph frequencies and matrices of $D$ and $I$ (according to Nei, 1972) and of Rogers' (1972) genetic distance were calculated 


\section{TABLE I}

Sampling localities and species distribution.

\begin{tabular}{|c|c|c|c|c|c|c|}
\hline $\begin{array}{l}\text { Station } \\
\text { no. }\end{array}$ & Species & Country & $\begin{array}{l}\text { Prov. } \\
\text { /dept. }\end{array}$ & $\begin{array}{l}\text { Drainage } \\
\text { system }\end{array}$ & $\begin{array}{l}\text { Locality } \\
\text { description }\end{array}$ & $\begin{array}{l}\text { Sampling } \\
\text { date }\end{array}$ \\
\hline 1 & G. stupendus & France & Var & Gapeau & $\begin{array}{l}\text { Source du Gapeau, alongside road } \\
\mathrm{D} 2,2 \mathrm{~km} \mathrm{~S} \text {. of Signes, } 40 \mathrm{~km} \mathrm{~N} \text {. of } \\
\text { Toulon }\end{array}$ & 29-VII-'86 \\
\hline 2 & G. orinos & France & Ardèche & Ardèche & $\begin{array}{l}\text { Rivulet draining into the Ardèche, at } \\
\text { crossing with road D } 533,4 \mathrm{~km} \mathrm{~N} . \mathrm{W} \text {. } \\
\text { of Vals-les-Bains }\end{array}$ & $\begin{array}{l}\text { 10-VII-'86 } \\
\text { 7-V-' } 86\end{array}$ \\
\hline 3 & G. cf. fossarum & France & Ariège & Ariège & $\begin{array}{l}\text { Confluent of the Ariège, } 0.5 \mathrm{~km} \mathrm{~N} \text {. of } \\
\text { Alliat, ca. } 10 \mathrm{~km} \mathrm{~W} \text {. of Tarascon }\end{array}$ & $\begin{array}{l}\text { 7-VIII-' } 87 \\
16-\mathrm{X}-' 87\end{array}$ \\
\hline 4 & G. cf. fossarum & France & Ariège & Ariège & $\begin{array}{l}\text { Tricklet alongside road D18, } 0.5 \mathrm{~km} \\
\text { W. of Suc, ca. } 20 \mathrm{~km} \mathrm{W.} \mathrm{of} \mathrm{Tarascon}\end{array}$ & $\begin{array}{l}\text { 7-V-'86 } \\
\text { 7-VIII-'87 }\end{array}$ \\
\hline 5 & G. cf. fossarum & France & Ariège & Garonne & $\begin{array}{l}\text { Rivulet crossing road D18, } 1.2 \mathrm{~km} \mathrm{E} \text {. } \\
\text { of Aulus-les-Bains, ca. } 30 \mathrm{~km} \mathrm{~W} \text {. of } \\
\text { Tarascon. }\end{array}$ & $7-V-' 86$ \\
\hline 6 & G. gauthieri & Spain & Cuenca & Tajo & $\begin{array}{l}\text { Río Albalate, alongside road N320 at } \\
\text { Arralba, ca. } 32 \mathrm{~km} \mathrm{N.N.W.} \mathrm{of} \\
\text { Cuenca. }\end{array}$ & $9-V-' 86$ \\
\hline 7 & G. gauthieri & Spain & Cuenca & Guadiana & $\begin{array}{l}\text { Río Cigüela, alongside road } \mathrm{N} 400 \text { at } \\
\text { Naharros, ca. } 35 \mathrm{~km} \mathrm{~W} \text {. of Cuenca. }\end{array}$ & $\begin{array}{l}10-V-' 86 \\
29-V-' 86\end{array}$ \\
\hline 8 & G. ibericus & Spain & Cuenca & Jucar & $\begin{array}{l}\text { Río Jucar, at its confluence with the } \\
\text { Laguna de Uña, ca. } 36 \mathrm{~km} \mathrm{~N} \text {. of } \\
\text { Cuenca }\end{array}$ & $\begin{array}{l}10-V-' 86 \\
29-I V-' 87\end{array}$ \\
\hline 9 & G. ibericus & Spain & Cuenca & Tajo & $\begin{array}{l}\text { Spring called Cañada de las Tablas, } \\
\text { draining into the Río Cuerbo, } 12 \mathrm{~km} \\
\text { N. of Tragacete }\end{array}$ & 28-IV-'87 \\
\hline 10 & G. gauthieri & Spain & Valladolid & Duero & $\begin{array}{l}\text { Río Cega, crossing road C112 near } \\
\text { Iscar, ca. } 12 \mathrm{~km} \mathrm{~W} \text {. of Cuéllar }\end{array}$ & $\begin{array}{l}13-V-' 86 \\
29-I V-' 87\end{array}$ \\
\hline 11 & G. gauthieri & Spain & Segovia & Duero & $\begin{array}{l}\text { Río Cerquilla, crossing road C601, } 2 \\
\text { km S. of Cuéllar }\end{array}$ & $12-V-' 86$ \\
\hline 12 & G. gauthieri & Spain & Valladolid & Duero & $\begin{array}{l}\text { Confluent of Río Pisuerga, crossing } \\
\text { road Va901, } 2 \mathrm{~km} \mathrm{~S} \text {. of Cigales }\end{array}$ & $13-V-' 86$ \\
\hline
\end{tabular}

TABLE II

Morphological differentiation of some taxonomically important characters in G. orinos, G. cf. fossarum, G. stupendus, G. ibericus, and G. gauthieri.

\begin{tabular}{lllllll}
\hline & G. orinos & G. cf. fossarum & G. stupendus & G. ibericus & G. gauthieri & G. gauthieri \\
Station & 2 & $3,4,5$ & 1 & 8,9 & 6,7 & $10,11,12$ \\
\hline Setosity A2 & moderate & moderate & moderate & moderate & little & little \\
Calceoli & + & - & - & - & + & + \\
Setosity P5-7 & long & moderate & long & long & absent & little \\
Setae longer than spines & ++ & + & ++ & ++ & - & - \\
Uropod 3: endop./exop. & $1 / 2$ & $1 / 2$ & $1 / 2$ & $2 / 3-3 / 4$ & $3 / 4$ & $3 / 4$ \\
Setae on dorsal surface telson & + & + & + & - & + & + \\
\hline
\end{tabular}



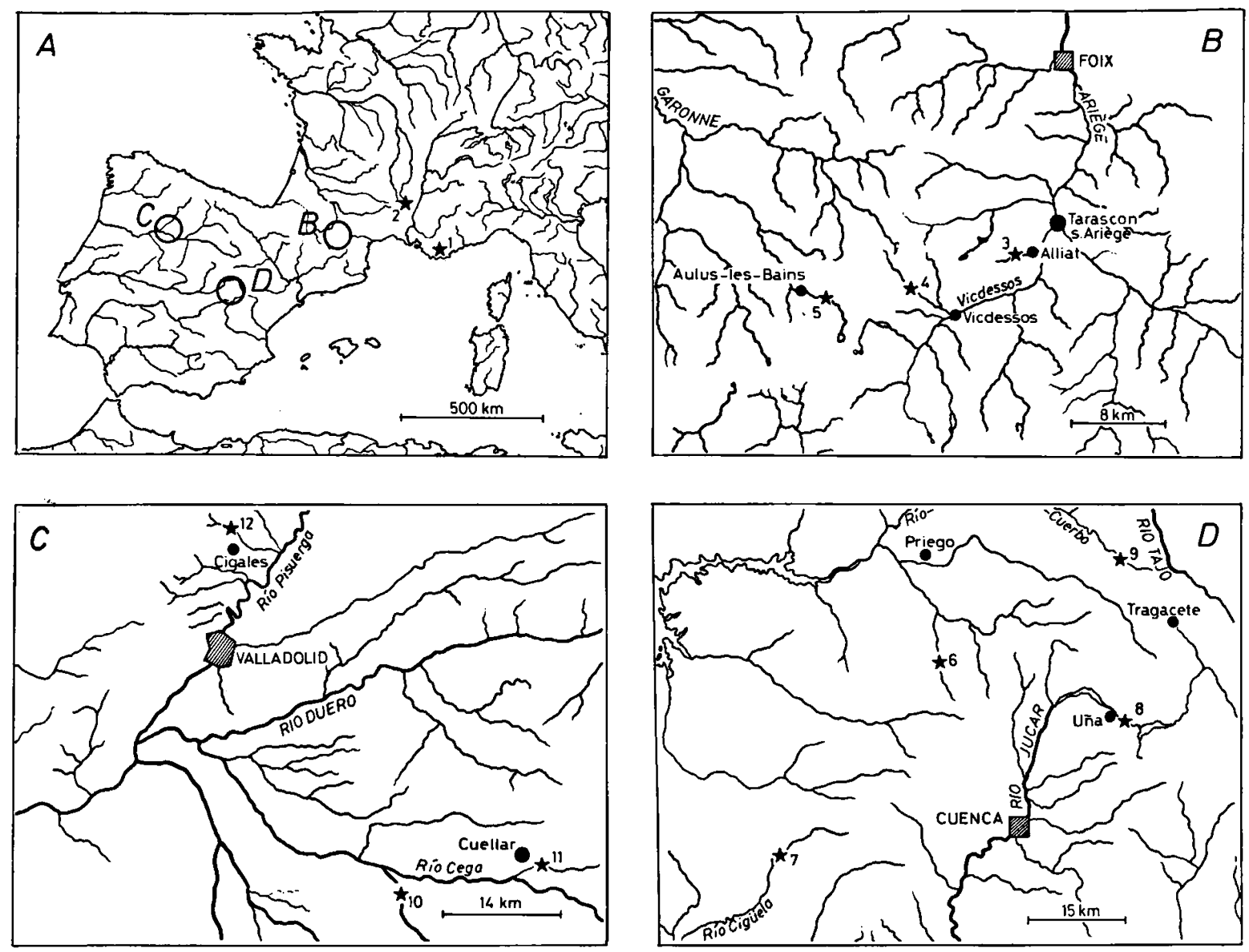

Fig. 1. A-D, Study areas and sampling localities (cf. table I).

with the computer program BIOSYS-1 (Swofford Selander, 1981). From these data, an UPGMA dendrogram (Sneath \&okal, 1973) of Nei's genetic distance (D) (Nei, 1972) and a distance Wagner network (Farris, 1972) based upon Rogers' genetic distance (Rogers, 1972) were constructed. A "character state" transformation scheme was calculated with the aid of the program Jelly (Ellis, 1987). A chi-square test was performed to test observed genotype frequencies and those expected under Hardy-Weinberg equilibrium. Electromorph frequencies of samples taken at stations 3, 4, 7, 8 and 10 (table I, figs. 1B-D) did not differ significantly in 1986 and 1987, thus these data are combined in the analysis.

Nei's $D$ has often been criticized because of its nonmetricity which makes it unsuitable for deriving phylogenetic trees. Moreover, both Nei's distance coefficient and the UPGMA method require the assumption of homogeneous evolutionary rates (see Farris, 1981; Swofford, 1981; Mickevich Miller, 1981; Buth, 1984; Rogers, 1986). Nevertheless, Nei's $D$ - and I-values (whether or not in combination with the UPGMA method) have been widely used by authors for studying speciation and for estimating divergence time (e.g. Thorpe, 1982; for Crustacea, among others, Sbordoni et al., 1980; Siegismund et al., 1985; Bert, 1986).

The Wagner distance procedure maximizes the information contained in the matrix of genetic distances and does not depend on the assumption of homogeneous evolutionary rates. This method results in the formation of the most parsimonious trees, i.e., trees with a minimum length.

Shortcomings of methods based upon distance matrices are the generation of "impossible ancestors" (Farris, 1981; Mickevich * Mitter, 1981; Swofford * Berlocher, 1987) and the neglection of less common electromorphs: a group of taxa can exhibit uniformly high pairwise genetic similarities and still possess a very different combination of electromorphs (Swofford Berlocher, 1987).

The Jelly program (Ellis, 1987) copes with these problems. This method, comparable to the ROGAD 


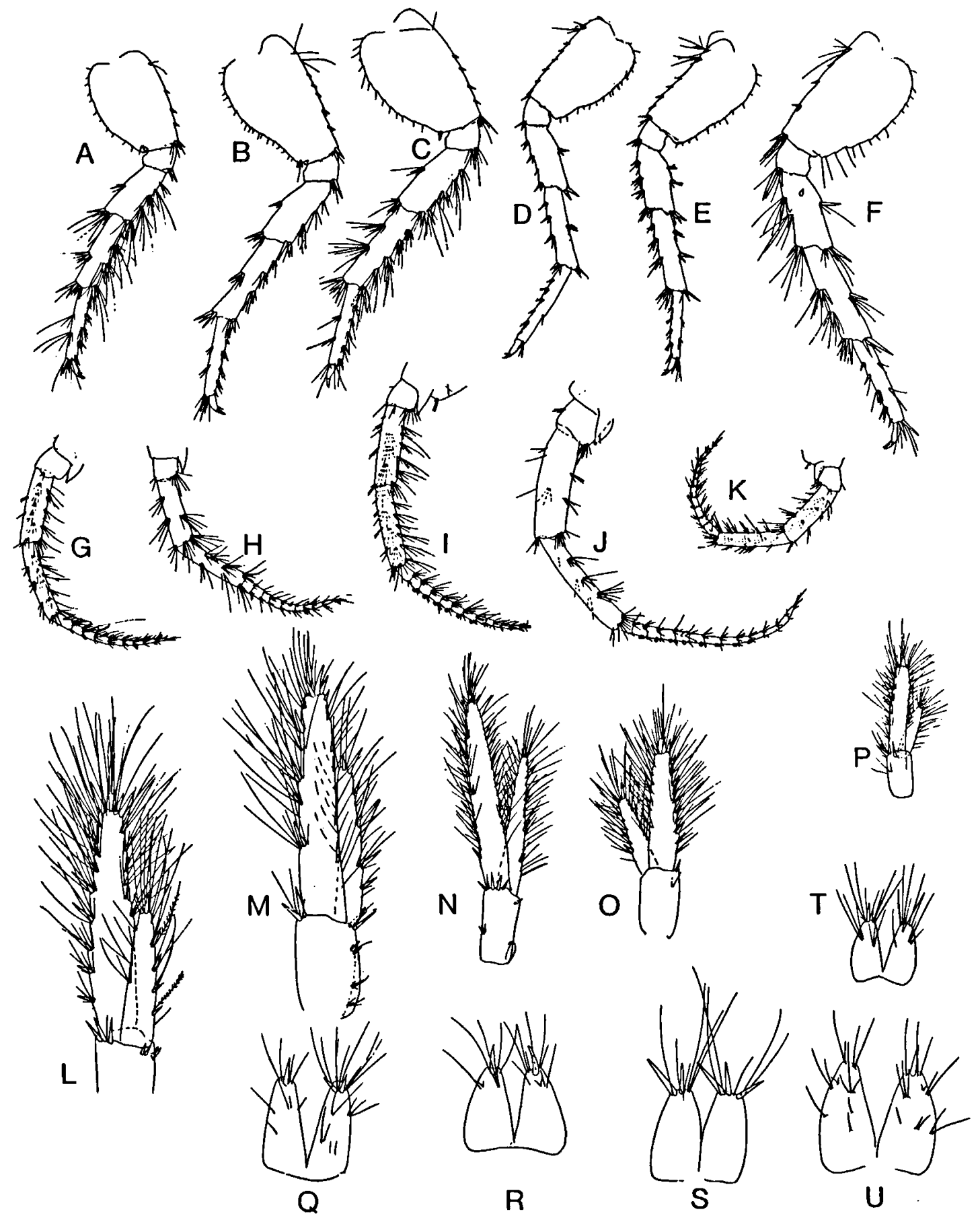

Fig. 2. Morphological differentiation of some taxonomically important characters in males of $G$. orinos (station 2), $G$. cf. fossarum (station 3), G. stupendus (station 1), G. ibericus (station 8) and G. gauthieri (stations 6+11).

A-F: pereiopod 7 of $G$. stupendus, G. cf. fossarum, G. orinos, G. gauthieri from station 11, G. gauthieri from station 6 , and G. ibericus, respectively;

G-K: antenna 2 of $G$. cf. fossarum, G. ibericus, G. orinos, G. gauthieri from station 6, and $G$. stupendus, respectively; L-P: uropod 3 of $G$. cf. fossarum, G. ibericus, G. gauthieri from station 6, G. orinos and $G$. stupendus, respectively; Q-U: telson of $G$. gauthieri from station $6, G$. cf. fossarum, G. ibericus, G. stupendus and $G$. orinos, respectively. 
TABLE III

Enzymes studied and buffer systems employed.

\begin{tabular}{lllll}
\hline Enzyme & Abbreviation & E.C. no. & Buffer system ${ }^{*}$ & Number of loci \\
\hline Adenosine deaminase & ADA & 3.5 .4 .4 & IV & 2 \\
Alkaline phosphatase & ALP & 3.1 .3 .1 & VI & 2 \\
Arginine phosphate kinase & APK & 2.7 .3 .3 & II, IV, V & 1 \\
Esterase & EST & 3.1 .1 .1 & III & 2 \\
Glutamate dehydrogenase & GDH & 1.1 .1 .47 & III & 1 \\
Glutamic oxaloacetic transaminase & GOT & 2.6 .1 .1 & I & 2 \\
Glucose phosphate isomerase & GPI & 5.3 .1 .11 & II, V & 1 \\
Hexokinase & HK & 2.7 .1 .1 & IV & 3 \\
Leucine aminopeptidase & LAP & 3.4 .1 .1 & IV & 1 \\
Malate dehydrogenase & MDH & 1.1 .1 .37 & I & 2 \\
Malic enzyme & ME & 1.1 .1 .40 & II, IV & 1 \\
Mannose phosphate isomerase & MPI & 5.3 .1 .8 & II & 1 \\
Peptidase & PEP & $3.4 .11 / 13$ & V & 5 \\
6 Phosphogluconate dehydrogenase & GPGD & 1.1 .1 .44 & VII & 1 \\
Pyruvate kinase & PK & 2.7 .1 .40 & II, V, VII & 1 \\
\hline
\end{tabular}

* The following buffer systems were used: (I) Amine citrate buffer pH 6.1 (Clayton \& Tretiak, 1972); (II) Tris maleate buffer pH 7.2 (Harris \& Hopkinson, 1976); (III) Tris citrate buffer pH 8.0 (Selander et al., 1971); (IV) Tris EDTA borate buffer pH 8.9 (Ayala et al., 1972; modification after Scholl et al., 1978); (V) phosphate buffer pH 6.7 (Selander et al., 1971); (VI) Tris citrate borate discontinuous buffer system pH 8.2-8.7 (Poulik, 1957); (VII) Histidine sodium citrate buffer (Brewer, 1970), with the addition of NADP ( $5 \mathrm{mg} / \mathrm{l}$ in gel, $10 \mathrm{mg} / \mathrm{l}$ in cathodal tray buffer).

method (Rogers, 1984; Swofford \& Berlocher, 1987), generates Wagner character states from elecromorph frequency characters using the HAP algorithm (Rogers, 1984). It considers frequencies of a particular electromorph as a character and any combination of electromorph frequencies as a particular character state. The frequency distributions ("character states") attributed to each of the HTU's (Hypothetical Taxonomic Units) are not selected from one of the "states" occurring in the OTU's (Operational Taxonomic Units), but have unique values of their own. These "character states" consist of arrays of electromorph frequencies in which the frequencies sum up to one, thus satisfying the "additivity requirement"' (see Swofford \& Berlocher, 1987). This method has also the advantages of the Hennigian method as proposed by Patton \& Avise (1983), but it copes with criticisms by Swofford \& Berlocher (1987) by considering all characters simultaneously.

The Jelly program starts by generating a user-defined number of networks, which are stocked in a so-called "arena". The concept of the arena is based on the model of Darwinian selection. The arena is an imaginary zone of memory, where a population of competing networks resides. Then, in a user-defined number of cycles, one of the networks is selected; the chance of being selected is maximal for the best network. Part of the OTU's is removed from the network and then refitted in an attempt to find a better network. The resulting network replaces the worst network in the arena. When no further improvement (viz. decrease in length) occurs in the residing networks, the program is stopped. The results obtained by the three methods discussed above are compared in order to evaluate differences due to the application of a particular method.

\section{Cross-breeding experiments}

Cross-breeding experiments followed Pinkster (1983) and were carried out (a) between morphologically similar but geographically distant $G$. gauthieri populations from the Valladolid area (station 10; fig. 1C) and the Serranía de Cuenca (station 7; fig. 1D); (b) between morphologically different but geographically neighbouring populations of G. ibericus (station 8; fig. 1D) and G. gauthieri (station 7; fig. 1D) and (c) between morphologically identical but genetically different populations of $G$. ibericus (stations 8 and 9 ; fig. 1D).

\section{RESULTS}

Twenty-one gene loci from seventeen enzyme systems were scorable in all populations. Elec- 
tromorph frequencies and relative mobilities of the enzymes coded for by these loci are given in table IV. Electromorphs of the Lap locus were monomorphic for all populations. Two or more loci were resolved in eight of the enzyme systems: ADA, ALP, EST, GOT, HK, MDH and both PEP stainings (table III). Among these, the locus that codes for the enzyme with the highest anodal mobility has been numbered 1. The gene products for two enzyme systems moved cathodically: GOT-2 and MDH-2. Enzyme products of the $P$ ep loci have been

\section{TABLE IV}

Electromorph frequency distribution at 20 enzyme loci. $\mathrm{N}=$ sample size; $h=$ heterozygosity per locus (direct count);

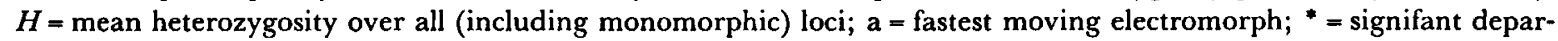
ture from Hardy-Weinberg distribution $(\mathrm{P}<0.05)$.

\begin{tabular}{|c|c|c|c|c|c|c|c|c|c|c|c|c|c|}
\hline \multirow{2}{*}{$\begin{array}{r}\text { Locus } \\
\text { ele }\end{array}$} & & \multicolumn{12}{|c|}{ Station no. } \\
\hline & electromorph & 1 & 2 & 3 & 4 & 5 & 6 & 7 & 8 & 9 & 10 & 11 & 12 \\
\hline \multirow[t]{3}{*}{ Ada-1 } & $\mathbf{a}$ & 0.00 & 0.00 & 0.00 & 0.00 & 0.00 & 0.00 & 0.00 & 0.00 & 1.00 & 0.00 & 0.00 & 0.00 \\
\hline & b & 1.00 & 1.00 & 1.00 & 1.00 & 1.00 & 1.00 & 1.00 & 1.00 & 0.00 & 1.00 & 1.00 & 1.00 \\
\hline & $\mathbf{N}$ & 99 & 34 & 20 & 37 & 27 & 25 & 28 & 47 & 32 & 30 & 47 & 34 \\
\hline \multirow[t]{4}{*}{$A l p-1$} & a & 0.00 & 0.00 & 0.00 & 0.00 & 0.00 & 1.00 & 1.00 & 0.00 & 0.00 & 0.00 & 0.00 & 0.00 \\
\hline & b & 1.00 & 1.00 & 1.00 & 1.00 & 1.00 & 0.00 & 0.00 & 1.00 & 1.00 & 0.00 & 0.00 & 0.00 \\
\hline & c & 0.00 & 0.00 & 0.00 & 0.00 & 0.00 & 0.00 & 0.00 & 0.00 & 0.00 & 1.00 & 1.00 & 1.00 \\
\hline & $\mathbf{N}$ & 99 & 47 & 50 & 50 & 40 & 45 & 33 & 28 & 26 & 45 & 59 & 48 \\
\hline \multirow[t]{4}{*}{ Alp-2 } & $\mathrm{a}$ & 1.00 & 1.00 & 1.00 & 1.00 & 1.00 & 0.61 & 0.70 & 1.00 & 1.00 & 1.00 & 1.00 & 1.00 \\
\hline & b & 0.00 & 0.00 & 0.00 & 0.00 & 0.00 & 0.39 & 0.30 & 0.00 & 0.00 & 0.00 & 0.00 & 0.00 \\
\hline & $h$ & 0.00 & 0.00 & 0.00 & 0.00 & 0.00 & 0.39 & 0.42 & 0.00 & 0.00 & 0.00 & 0.00 & 0.00 \\
\hline & $\mathbf{N}$ & 99 & 47 & 50 & 50 & 40 & 41 & 33 & 59 & 45 & 45 & 59 & 48 \\
\hline \multirow[t]{4}{*}{$A p k$} & $\mathbf{a}$ & 0.00 & 0.00 & 0.00 & 0.00 & 0.00 & 1.00 & 1.00 & 1.00 & 1.00 & 0.96 & 0.96 & 0.97 \\
\hline & b & 1.00 & 1.00 & 1.00 & 1.00 & 1.00 & 0.00 & 0.00 & 0.00 & 0.00 & 0.04 & 0.04 & 0.03 \\
\hline & $h$ & 0.00 & 0.00 & 0.00 & 0.00 & 0.00 & 0.00 & 0.00 & 0.00 & 0.00 & 0.08 & 0.08 & 0.05 \\
\hline & $\mathbf{N}$ & 99 & 50 & 45 & 53 & 42 & 57 & 38 & 81 & 33 & 39 & 51 & 39 \\
\hline \multirow[t]{4}{*}{ Est-1 } & $\mathbf{a}$ & 0.00 & 0.00 & 0.00 & 0.00 & 0.00 & 0.00 & 0.00 & 0.00 & 0.00 & 1.00 & 1.00 & 1.00 \\
\hline & b & 0.00 & 0.00 & 0.00 & 0.00 & 0.00 & 1.00 & 1.00 & 1.00 & 1.00 & 0.00 & 0.00 & 0.00 \\
\hline & c & 1.00 & 1.00 & 1.00 & 1.00 & 1.00 & 0.00 & 0.00 & 0.00 & 0.00 & 0.00 & 0.00 & 0.00 \\
\hline & $\mathbf{N}$ & 99 & 8 & 16 & 27 & 33 & 18 & 28 & 51 & 51 & 38 & 22 & 17 \\
\hline \multirow[t]{3}{*}{ Est-2 } & $\mathbf{a}$ & 0.00 & 0.00 & 0.00 & 0.00 & 0.00 & 1.00 & 1.00 & 1.00 & 0.00 & 1.00 & 1.00 & 1.00 \\
\hline & b & 1.00 & 1.00 & 1.00 & 1.00 & 1.00 & 0.00 & 0.00 & 0.00 & 1.00 & 0.00 & 0.00 & 0.00 \\
\hline & $\mathbf{N}$ & 99 & 8 & 16 & 27 & 33 & 18 & 28 & 51 & 51 & 38 & 22 & 17 \\
\hline \multirow[t]{3}{*}{$G d h$} & $\mathbf{a}$ & 1.00 & 1.00 & 1.00 & 1.00 & 1.00 & 1.00 & 1.00 & 1.00 & 1.00 & 0.00 & 0.00 & 0.00 \\
\hline & b & 0.00 & 0.00 & 0.00 & 0.00 & 0.00 & 0.00 & 0.00 & 0.00 & 0.00 & 1.00 & 1.00 & 1.00 \\
\hline & $\mathbf{N}$ & 99 & 71 & 58 & 59 & 94 & 82 & 57 & 80 & 57 & 70 & 63 & 33 \\
\hline \multirow[t]{6}{*}{ Got-1 } & $\mathbf{a}$ & 0.00 & 0.01 & 1.00 & 1.00 & 1.00 & 0.00 & 0.00 & 0.00 & 1.00 & 0.00 & 0.00 & 0.00 \\
\hline & b & 0.00 & 0.00 & 0.00 & 0.00 & 0.00 & 1.00 & 1.00 & 1.00 & 0.00 & 1.00 & 1.00 & 1.00 \\
\hline & c & 0.90 & 0.99 & 0.00 & 0.00 & 0.00 & 0.00 & 0.00 & 0.00 & 0.00 & 0.00 & 0.00 & 0.00 \\
\hline & d & 0.10 & 0.00 & 0.00 & 0.00 & 0.00 & 0.00 & 0.00 & 0.00 & 0.00 & 0.00 & 0.00 & 0.00 \\
\hline & $h$ & 0.20 & 0.14 & 0.00 & 0.00 & 0.00 & 0.00 & 0.00 & 0.00 & 0.00 & 0.00 & 0.00 & 0.00 \\
\hline & $\mathbf{N}$ & 99 & 71 & 29 & 44 & 77 & 57 & 44 & 74 & 70 & 44 & 45 & 29 \\
\hline \multirow[t]{5}{*}{ Got-2 } & $a$ & 0.00 & 0.00 & 0.00 & 0.00 & 0.00 & 1.00 & 1.00 & 1.00 & 0.55 & 1.00 & 1.00 & 1.00 \\
\hline & b & 1.00 & 1.00 & 1.00 & 1.00 & 1.00 & 0.00 & 0.00 & 0.00 & 0.00 & 0.00 & 0.00 & 0.00 \\
\hline & c & 0.00 & 0.00 & 0.00 & 0.00 & 0.00 & 0.00 & 0.00 & 0.00 & 0.45 & 0.00 & 0.00 & 0.00 \\
\hline & $h$ & 0.00 & 0.00 & 0.00 & 0.00 & 0.00 & 0.00 & 0.00 & 0.00 & 0.50 & 0.00 & 0.00 & 0.00 \\
\hline & $\mathbf{N}$ & 99 & 54 & 29 & 37 & 62 & 47 & 40 & 57 & 54 & 64 & 33 & 39 \\
\hline
\end{tabular}


Table IV (continuation)

\begin{tabular}{|c|c|c|c|c|c|c|c|c|c|c|c|c|c|}
\hline \multirow{2}{*}{\multicolumn{2}{|c|}{$\begin{array}{l}\text { Locus } \\
\text { electromorph }\end{array}$}} & \multicolumn{12}{|c|}{ Station no. } \\
\hline & & \multirow{2}{*}{$\frac{1}{0.00}$} & \multirow{2}{*}{$\frac{2}{0.27}$} & \multirow{2}{*}{$\frac{3}{0.00}$} & \multirow{2}{*}{$\begin{array}{c}4 \\
0.00\end{array}$} & \multirow{2}{*}{$\begin{array}{c}5 \\
0.00\end{array}$} & \multirow{2}{*}{\begin{tabular}{c|}
6 \\
0.00
\end{tabular}} & \multirow{2}{*}{$\begin{array}{c}7 \\
0.00\end{array}$} & \multirow{2}{*}{$\frac{8}{0.00}$} & \multirow{2}{*}{$\frac{9}{0.00}$} & \multirow{2}{*}{$\frac{10}{0.00}$} & \multirow{2}{*}{$\frac{11}{0.00}$} & \multirow{2}{*}{$\begin{array}{c}12 \\
0.00\end{array}$} \\
\hline$G p i$ & a & & & & & & & & & & & & \\
\hline & b & 0.01 & 0.66 & 0.00 & 0.00 & 0.00 & 0.00 & 0.00 & 0.00 & 0.00 & 0.00 & 0.00 & 0.00 \\
\hline & c & 0.95 & 0.00 & 0.00 & 0.00 & 0.00 & 0.00 & 0.00 & 0.00 & 0.00 & 0.00 & 0.00 & 0.00 \\
\hline & d & 0.01 & 0.07 & 0.00 & 0.00 & 0.00 & 0.00 & 0.00 & 0.00 & 0.00 & 0.00 & 0.00 & 0.00 \\
\hline & e & 0.03 & 0.00 & 0.00 & 0.00 & 0.00 & 0.00 & 0.00 & 0.67 & 0.00 & 0.00 & 0.00 & 0.00 \\
\hline & f & 0.00 & 0.00 & 0.49 & 0.00 & 0.00 & 0.01 & 0.00 & 0.00 & 0.00 & 0.00 & 0.00 & 0.00 \\
\hline & $\mathbf{g}$ & 0.00 & 0.00 & 0.00 & 0.18 & 0.19 & 0.00 & 0.00 & 0.00 & 0.00 & 0.00 & 0.00 & 0.00 \\
\hline & $\mathrm{h}$ & 0.00 & 0.00 & 0.00 & 0.82 & 0.81 & 0.00 & 0.36 & 0.32 & 0.99 & 0.01 & 0.00 & 0.00 \\
\hline & i & 0.00 & 0.00 & 0.00 & 0.00 & 0.00 & 0.00 & 0.64 & 0.01 & 0.01 & 0.95 & 0.74 & 0.99 \\
\hline & $\mathrm{j}$ & 0.00 & 0.00 & 0.51 & 0.00 & 0.00 & 0.00 & 0.00 & 0.00 & 0.00 & 0.04 & 0.26 & 0.01 \\
\hline & k & 0.00 & 0.00 & 0.00 & 0.00 & 0.00 & 0.99 & 0.00 & 0.00 & 0.00 & 0.00 & 0.00 & 0.00 \\
\hline & $h$ & 0.07 & 0.42 & 0.47 & 0.25 & 0.24 & 0.02 & 0.40 & 0.49 & 0.02 & 0.10 & 0.44 & 0.02 \\
\hline & $\mathbf{N}$ & 99 & $48^{*}$ & 59 & 91 & 70 & 79 & 70 & 119 & 52 & 51 & 50 & 45 \\
\hline \multirow[t]{5}{*}{$H k-1$} & $\mathbf{a}$ & 0.52 & 1.00 & 1.00 & 1.00 & 1.00 & 0.00 & 0.00 & 0.00 & 0.00 & 0.00 & 0.00 & 0.00 \\
\hline & b & 0.48 & 0.00 & 0.00 & 0.00 & 0.00 & 1.00 & 1.00 & 1.00 & 0.00 & 1.00 & 1.00 & 1.00 \\
\hline & c & 0.00 & 0.00 & 0.00 & 0.00 & 0.00 & 0.00 & 0.00 & 0.00 & 1.00 & 0.00 & 0.00 & 0.00 \\
\hline & $h$ & 0.46 & 0.00 & 0.00 & 0.00 & 0.00 & 0.00 & 0.00 & 0.00 & 0.00 & 0.00 & 0.00 & 0.00 \\
\hline & $\mathbf{N}$ & 37 & 59 & 38 & 42 & 78 & 38 & 28 & 62 & 67 & 38 & 67 & 32 \\
\hline \multirow[t]{7}{*}{$M d h-1$} & $\mathbf{a}$ & 1.00 & 0.90 & 0.00 & 0.00 & 0.00 & 0.00 & 0.00 & 0.00 & 0.00 & 0.00 & 0.00 & 0.00 \\
\hline & b & 0.00 & 0.10 & 1.00 & 1.00 & 1.00 & 0.00 & 0.00 & 1.00 & 1.00 & 0.00 & 0.00 & 0.00 \\
\hline & c & 0.00 & 0.00 & 0.00 & 0.00 & 0.00 & 1.00 & 1.00 & 0.00 & 0.00 & 0.00 & 0.00 & 0.00 \\
\hline & d & 0.00 & 0.00 & 0.00 & 0.00 & 0.00 & 0.00 & 0.00 & 0.00 & 0.00 & 1.00 & 0.97 & 1.00 \\
\hline & e & 0.00 & 0.00 & 0.00 & 0.00 & 0.00 & 0.00 & 0.00 & 0.00 & 0.00 & 0.00 & 0.03 & 0.00 \\
\hline & $h$ & 0.00 & 0.16 & 0.00 & 0.00 & 0.00 & 0.00 & 0.00 & 0.00 & 0.00 & 0.00 & 0.05 & 0.00 \\
\hline & $\mathbf{N}$ & 99 & 55 & 41 & 61 & 60 & 77 & 50 & 54 & 15 & 49 & 61 & 39 \\
\hline$M d h-2$ & $\mathbf{a}$ & 0.00 & 0.00 & 0.74 & 0.64 & 0.48 & 0.00 & 0.00 & 0.00 & 0.00 & 0.00 & 0.00 & 0.00 \\
\hline & b & 1.00 & 1.00 & 0.26 & 0.36 & 0.52 & 1.00 & 1.00 & 1.00 & 1.00 & 1.00 & 1.00 & 1.00 \\
\hline & $h$ & 0.00 & 0.00 & 0.32 & 0.37 & 0.54 & 0.00 & 0.00 & 0.00 & 0.00 & 0.00 & 0.00 & 0.00 \\
\hline & $\mathbf{N}$ & 99 & 54 & 31 & 40 & 44 & 66 & 50 & 58 & 19 & 49 & 63 & 51 \\
\hline Me & $\mathbf{a}$ & 0.00 & 0.00 & 0.00 & 0.00 & 0.00 & 0.00 & 0.00 & 1.00 & 1.00 & 0.00 & 0.00 & 0.00 \\
\hline & b & 1.00 & 1.00 & 1.00 & 1.00 & 1. & 1. & 1.00 & 0.00 & 0.00 & 0.00 & 0.00 & 0.00 \\
\hline & c & 0.00 & 0.00 & 0.00 & 0.00 & 0.00 & 0.00 & 0.00 & 0.00 & 0.00 & 1.00 & 1.00 & 1.00 \\
\hline & $\mathbf{N}$ & 99 & 62 & 35 & 72 & 85 & 76 & 56 & 80 & 22 & 70 & 74 & 31 \\
\hline$M p i$ & a & 0.00 & 0.00 & 0.00 & 0.00 & 0.00 & 1 & $1 . c$ &  & 0.00 & 0.0 & 0.00 & 0.00 \\
\hline & b & 0.03 & 0.00 & 0.00 & 0.00 & 0.00 & 0.00 & 0.00 & 0.87 & 0.13 & 0.00 & 0.00 & 0.00 \\
\hline & c & 0.90 & 0.00 & 0.00 & 0.00 & 0.00 & 0.00 & 0.00 & 0.12 & 0.87 & 0.02 & 0.00 & 0.01 \\
\hline & d & 0.07 & 1.00 & 0.00 & 0.00 & 0.00 & 0.00 & 0.00 & 0.01 & 0.00 & 0.00 & 0.00 & 0.00 \\
\hline & e & 0.00 & 0.00 & 1.00 & 1.00 & 0.97 & 0.00 & 0.00 & 0.00 & 0.00 & 0.00 & 0.00 & 0.00 \\
\hline & f & 0.00 & 0.00 & 0.00 & 0.00 & 0.03 & 0.00 & 0.00 & 0.00 & 0.00 & 0.31 & 0.00 & 0.41 \\
\hline & g & 0.00 & 0.00 & 0.00 & 0.00 & 0.00 & 0.00 & 0.00 & 0.00 & 0.00 & 0.67 & 1.00 & 0.58 \\
\hline & $h$ & 0.12 & 0.00 & 0.00 & 0.00 & 0.22 & 0.00 & 0.00 & 0.12 & 0.13 & 0.37 & 0.00 & 0.41 \\
\hline & $\mathbf{N}$ & 99 & 77 & 38 & 53 & 46 & 53 & 41 & $64^{*}$ & 35 & 46 & 53 & 37 \\
\hline Pep-2 & a & 0.00 & 0.00 & 0.00 & 0.00 & 0.00 & 0.00 & 0.00 & 0.00 & 0.92 & 0.00 & 0.00 & 0.00 \\
\hline & b & 0.00 & 0.00 & 0.00 & 0.00 & 0.00 & 0.00 & 0.00 & 0.00 & 0.00 & 0.48 & 0.00 & 0.00 \\
\hline & c & 0.00 & 0.00 & 0.00 & 0.00 & 0.00 & 1.00 & 1.00 & 1.00 & 0.08 & 0.00 & 0.00 & 0.00 \\
\hline & d & 0.00 & 0.00 & 0.00 & 0.00 & 0.00 & 0.00 & 0.00 & 0.00 & 0.00 & 0.52 & 1.00 & 1.00 \\
\hline & e & 1.00 & 0.00 & 1.00 & 1.00 & 1.00 & 0.00 & 0.00 & 0.00 & 0.00 & 0.00 & 0.00 & 0.00 \\
\hline & f & 0.00 & 1.00 & 0.00 & 0.00 & 0.00 & 0.00 & 0.00 & 0.00 & 0.00 & 0.00 & 0.00 & 0.00 \\
\hline & $h$ & 0.00 & 0.00 & 0.00 & 0.00 & 0.00 & 0.00 & 0.00 & 0.00 & 0.16 & 0.38 & 0.00 & 0.00 \\
\hline & $\mathbf{N}$ & 99 & 32 & 27 & 38 & 28 & 19 & 48 & 32 & 64 & 48 & 18 & 36 \\
\hline
\end{tabular}


Table IV (continuation)

\begin{tabular}{|c|c|c|c|c|c|c|c|c|c|c|c|c|c|}
\hline \multirow{2}{*}{\multicolumn{2}{|c|}{$\begin{array}{l}\text { Locus } \\
\quad \text { electromorph }\end{array}$}} & \multicolumn{12}{|c|}{ Station no. } \\
\hline & & \multirow{2}{*}{$\frac{1}{0.00}$} & \multirow{2}{*}{$\frac{2}{0.00}$} & \multirow{2}{*}{$\frac{3}{0.00}$} & \multirow{2}{*}{$\frac{4}{0.00}$} & \multirow{2}{*}{$\frac{5}{0.00}$} & \multirow{2}{*}{$\frac{6}{1.00}$} & \multirow{2}{*}{$\frac{7}{1.00}$} & \multirow{2}{*}{$\frac{8}{1.00}$} & \multirow{2}{*}{$\frac{9}{1.00}$} & \multirow{2}{*}{$\frac{10}{1.00}$} & \multirow{2}{*}{$\frac{11}{1.00}$} & \multirow{2}{*}{$\frac{12}{1.00}$} \\
\hline Pep-3 & a & & & & & & & & & & & & \\
\hline & b & 1.00 & 1.00 & 1.00 & 1.00 & 1.00 & 0.00 & 0.00 & 0.00 & 0.00 & 0.00 & 0.00 & 0.00 \\
\hline &  & 99 & 32 & 27 & 38 & 28 & 19 & 48 & 32 & 55 & 49 & 18 & 36 \\
\hline \multirow[t]{7}{*}{ Pep-4 } & $\mathbf{a}$ & 1.00 & 0.00 & 0.00 & 0.00 & 0.00 & 1.00 & 1.00 & 1.00 & 1.00 & 0.79 & 1.00 & 0.73 \\
\hline & b & 0.00 & 0.00 & 0.00 & 0.00 & 0.00 & 0.00 & 0.00 & 0.00 & 0.00 & 0.00 & 0.00 & 0.00 \\
\hline & c & 0.00 & 0.00 & 0.00 & 0.00 & 0.00 & 0.00 & 0.00 & 0.00 & 0.00 & 0.21 & 0.00 & 0.27 \\
\hline & d & 0.00 & 1.00 & 0.00 & 0.00 & 0.00 & 0.00 & 0.00 & 0.00 & 0.00 & 0.00 & 0.00 & 0.00 \\
\hline & e & 0.00 & 0.00 & 1.00 & 1.00 & 1.00 & 0.00 & 0.00 & 0.00 & 0.00 & 0.00 & 0.00 & 0.00 \\
\hline & $h$ & 0.00 & 0.00 & 0.00 & 0.00 & 0.00 & 0.00 & 0.00 & 0.00 & 0.00 & 0.31 & 0.00 & 0.33 \\
\hline & N & 99 & 32 & 27 & 38 & 28 & 19 & 48 & 32 & 55 & 49 & 18 & 30 \\
\hline \multirow[t]{3}{*}{ 6-Pgd } & $\mathbf{a}$ & 1.00 & 1.00 & 1.00 & 1.00 & 1.00 & 0.00 & 0.00 & 0.00 & 0.00 & 0.00 & 0.00 & 0.00 \\
\hline & b & 0.00 & 0.00 & 0.00 & 0.00 & 0.00 & 1.00 & 1.00 & 1.00 & 1.00 & 1.00 & 1.00 & 1.00 \\
\hline & $\mathbf{N}$ & 99 & 49 & 41 & 36 & 25 & 37 & 35 & 48 & 31 & 53 & 52 & 39 \\
\hline \multirow[t]{5}{*}{$P k$} & $\mathbf{a}$ & 0.00 & 0.00 & 0.00 & 0.00 & 0.00 & 1.00 & 1.00 & 1.00 & 1.00 & 0.00 & 0.00 & 0.81 \\
\hline & b & 1.00 & 1.00 & 1.00 & 1.00 & 1.00 & 0.00 & 0.00 & 0.00 & 0.00 & 1.00 & 1.00 & 0.19 \\
\hline & $h$ & 0.00 & 0.00 & 0.00 & 0.00 & 0.00 & 0.00 & 0.00 & 0.00 & 0.00 & 0.00 & 0.00 & 0.31 \\
\hline & $\mathrm{N}$ & 99 & 34 & 39 & 27 & 25 & 21 & 33 & 61 & 73 & 38 & 38 & 29 \\
\hline & H & 0.01 & 0.06 & 0.04 & 0.03 & 0.04 & 0.02 & 0.04 & 0.03 & 0.03 & 0.06 & 0.03 & 0.05 \\
\hline
\end{tabular}

numbered 1-2 (substrate 1-leucyl-glycyl-glycine), and 3-4 (substrate 1-leucyl-glycyltyrosine). The first stain (with 1-leucyl-glycylglycine as substrate) gives two zones of activity, the second (substrate 1-leucyl-glycyl-tyrosine) three. The first system in both stainings was identical (Pep-1). In HK and EST stains, three and four bands were observed, respectively. The Pep- 1 band was not consistently scorable in most populations and has therefore been omitted. In the ADA and HK stain only the first (fastest) band could be scored satisfactorily; in the EST stain the first and the second.

Five polymorphic loci, $A p k, H k-1, M p i, P e p-$ 2 , and $P k$ showed two bands in heterozygous individuals and were considered as monomeric enzymes. In six loci, Got-1, Got-2, Gpi, Mdh-1, $M d h-2$ and Pep-4 heterozygous individuals had three bands and these enzymes were considered as dimeric enzymes. No intra-population variation was noted in the remaining loci (table IV). The Alp-2 locus was polymorphic for the two $G$. gauthieri populations from stations $6+7$ (fig. 2D). The subunit structure at this locus could not be established, but for prawns it is referred to as monomeric (e.g. Mulley \& Latter, 1980).

\section{Genetic variation among populations}

Table IV shows considerable differences in allelic distribution among the populations studied. At a number of loci, the occurrence of specific electromorphs allows nearly complete discrimination between the French and the Iberian populations studied (viz., Apk, Est-1, Est-2, Got-1, Got-2, Pep-3 and 6-Pgd). At other loci, the occurrence of unique electromorphs is restricted to the populations from the Iberian peninsula: $A l p-1^{a, c}, A l p-2^{a}, E s t-1^{a, b}, G d h^{b}, M e^{a, d}$ and $P k^{b}$. G. ibericus from station 9 (tables I and IV; fig. 1D) deviates from this general pattern at the Est-2, Got-1 and $H k-1$ loci. The monomorphic Est-2 and Got-1 loci of G. ibericus from station 9 are fixed for electromorphs that characterize the French forms. Also, in this sample electromorphs occur (Ada- $1^{a}, H k-1^{c}$ and Got- $\left.2^{a}\right)$ that have not been found in any of the other populations. 
Differences between numbers of loci that are discriminative or diagnostic (i.e. there is no overlap of the allelic complements at the 0.99 level; see Ayala \& Powell, 1972) are summarized in table V. From the data in tables VI and VII, respectively, an UPGMA dendrogram and a distance Wagner network have been generated (figs. 3, 4, 6). From the data in table VI mean inter- and intra-area values of $I$ can be calculated. These values are shown in table VIII. Samples from French and Iberian populations are differentiated by a high number of diagnostic loci (table $\mathrm{V}$ ) and low inter-area values of $I$ (table VIII). The UPGMA dendrogram (fig. 3) and the distance Wagner network (figs. 4,6 ) show a high level of divergence between French and Iberian population samples. Interspecific differences among French populations are much smaller than interspecific or in some cases even intraspecific differences in the Iberian peninsula (tables V and VIII). The UPGMA dendrogram and the distance Wagner network show clearly the higher levels of divergence among the Spanish populations compared to the French ones. As expected, highest $I$-values are found among intra-areal populations of $G$. gauthieri. Inter-areal intraspecific $I$-values of $G$. gauthieri populations are much lower and exhibit no overlap with intraareal intra-specific values within this species. Moreover, inter-areal $I$-values of $G$. gauthieri populations are lower than intra-areal interspecific values of $I$ among populations of $G$. gauthieri and $G$. ibericus. There is also a considerable divergence between the two populations of $G$. ibericus (stations 8 and 9: $I=0.70$ ).

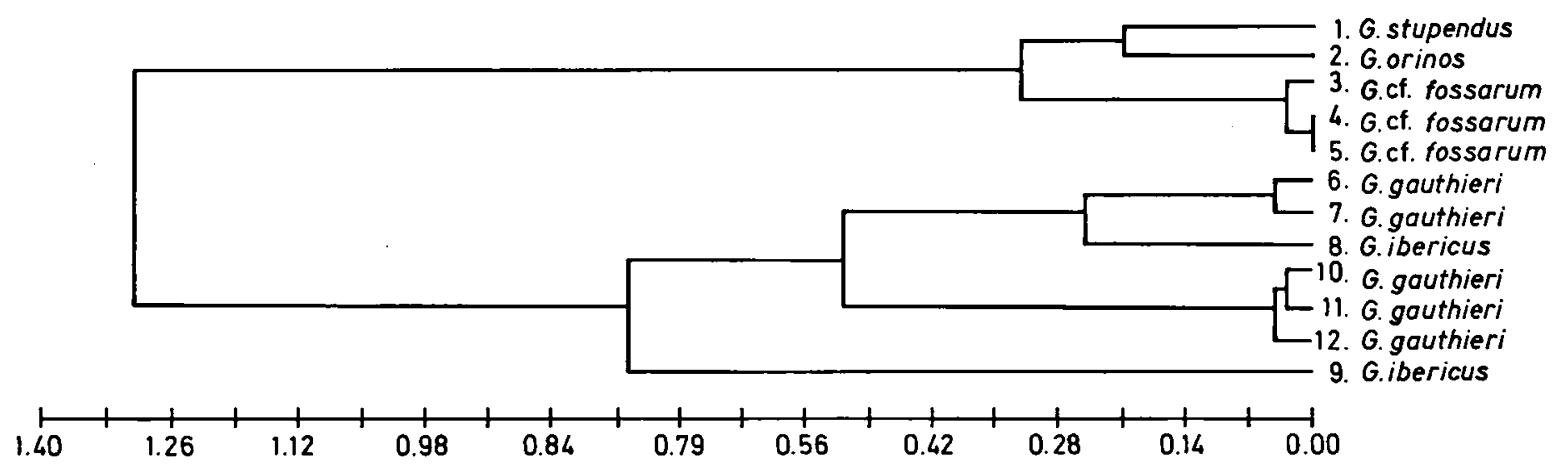

Fig. 3. UPGMA dendrogram of Nei's genetic distance $(D)$ based upon 21 enzyme loci.

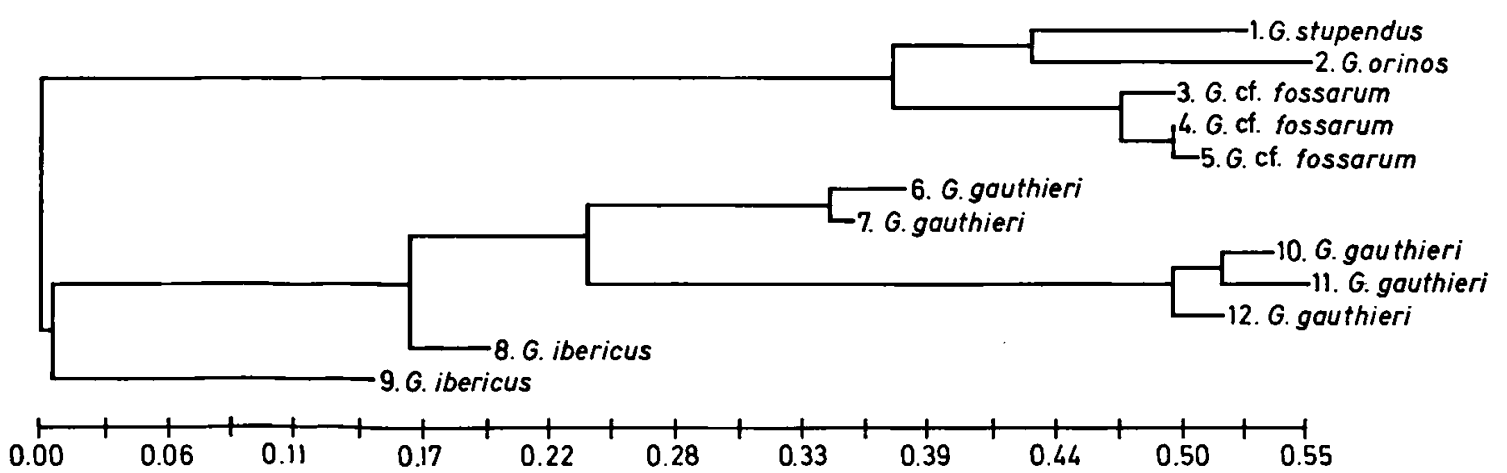

Fig. 4. Distance Wagner dendrogram of Rogers' genetic distance rooted at the midpoint of the longest path (Farris, 1972), based upon 21 enzyme loci. 
TABLE V

Number of diagnostic loci among samples of the populations studied (for further explanations see text).

\begin{tabular}{|c|c|c|c|c|c|c|c|c|c|c|c|c|}
\hline $\begin{array}{l}\text { Station no. } \\
\text { species }\end{array}$ & 1 & 2 & 3 & 4 & 5 & 6 & 7 & 8 & 9 & 10 & 11 & 12 \\
\hline $1 G$. stupendus & - & & & & & & & & & & & \\
\hline 2 G. orinos & 2 & - & & & & & & & & & & \\
\hline $3 G$. cf. fossarum & 5 & 4 & - & & & & & & & & & \\
\hline 4 G. cf. fossarum & 5 & 4 & 1 & - & & & & & & & & \\
\hline 5 G. cf. fossarum & 5 & 4 & 1 & - & - & & & & & & & \\
\hline 6 G. gauthieri & 14 & 17 & 14 & 15 & 15 & - & & & & & & \\
\hline 7 G. gauthieri & 14 & 17 & 15 & 14 & 14 & 1 & - & & & & & \\
\hline 8 G. ibericus & 11 & 14 & 12 & 11 & 11 & 5 & 4 & - & & & & \\
\hline 9 G. ibericus & 12 & 14 & 13 & 12 & 12 & 9 & 8 & 4 & - & & & \\
\hline 10 G. gauthieri & 12 & 15 & 14 & 15 & 14 & 8 & 7 & 12 & - & - & & \\
\hline 11 G. gauthieri & 13 & 15 & 14 & 15 & 14 & 8 & 7 & 7 & 11 & - & - & \\
\hline 12 G. gauthieri & 12 & 15 & 14 & 15 & 14 & 8 & 7 & 8 & 12 & - & - & - \\
\hline
\end{tabular}

TABLE VI

Matrix of genetic similarity and distance coefficients. Below diagonal: Nei's (1972) genetic distance. Above diagonal: Nei's (1972) genetic identity.

\begin{tabular}{lcccccccccccccc}
\hline $\begin{array}{l}\text { Station no. } \\
\text { species }\end{array}$ & 1 & 2 & 3 & 4 & 5 & 6 & 7 & 8 & 9 & 10 & 11 & 12 \\
\hline 1 G. stupendus & - & 0.80 & 0.74 & 0.74 & 0.75 & 0.35 & 0.36 & 0.38 & 0.39 & 0.32 & 0.32 & 0.27 \\
2 G. orinos & 0.22 & - & 0.70 & 0.71 & 0.72 & 0.27 & 0.28 & 0.30 & 0.30 & 0.25 & 0.25 & 0.21 \\
3 G. cf. fossarum & 0.31 & 0.35 & - & 0.97 & 0.97 & 0.24 & 0.25 & 0.31 & 0.36 & 0.22 & 0.22 & 0.18 \\
4 & G. cf. fossarum & 0.30 & 0.35 & 0.03 & - & 1.00 & 0.24 & 0.27 & 0.33 & 0.41 & 0.22 & 0.22 & 0.18 \\
5 G. cf. fossarum & 0.28 & 0.33 & 0.03 & 0.00 & - & 0.25 & 0.27 & 0.34 & 0.41 & 0.23 & 0.23 & 0.19 \\
6 G. gauthieri & 1.05 & 1.30 & 1.43 & 1.41 & 1.38 & - & 0.96 & 0.76 & 0.50 & 0.57 & 0.57 & 0.60 \\
7 G. gauthieri & 1.03 & 1.28 & 1.40 & 1.33 & 1.30 & 0.04 & - & 0.78 & 0.53 & 0.61 & 0.60 & 0.55 \\
8 & G. ibericus & 0.97 & 1.21 & 1.17 & 1.12 & 1.09 & 0.27 & 0.25 & - & 0.70 & 0.50 & 0.59 & 0.63 \\
9 & G. ibericus & 0.95 & 1.21 & 1.02 & 0.90 & 0.88 & 0.69 & 0.63 & 0.36 & - & 0.37 & 0.37 & 0.42 \\
10 & G. gauthieri & 1.14 & 1.38 & 1.52 & 1.51 & 1.47 & 0.57 & 0.50 & 0.53 & 0.99 & - & 0.98 & 0.95 \\
11 & G. gauthieri & 1.14 & 1.41 & 1.52 & 1.53 & 1.49 & 0.57 & 0.51 & 0.53 & 0.99 & 0.02 & - & 0.95 \\
12 & G. gauthieri & 1.30 & 1.57 & 1.74 & 1.72 & 1.70 & 0.51 & 0.44 & 0.47 & 0.90 & 0.05 & 0.05 & - \\
\hline
\end{tabular}

Main clusters in the UPGMA dendrogram and the distance Wagner network coincide with major geographical areas, although one conspicuous difference is the placement of $G$. ibericus from station 8. According to UPGMA, G. ibericus from station 8 has been clustered with G. gauthieri from stations 6 and 7 in the same geographic area. According to the Wagner network, $G$. ibericus from station 9 appears more related to $G$. ibericus from station 8 . However, the sample from station 9 is not clustered closely to the morphologically identical $G$. ibericus from station 8 nor with other populations from stations in the same area.

The 30 shortest ("best") trees generated by the Jelly program were almost identical (e.g. same length, symmetry, consensus, and topology). The network of fig. 5 has been selected for being optimal. The topology of the Jelly network (fig. 5) is rather similar to the 


\section{TABle VII}

Matrix of Rogers' (1972) genetic distance.

\begin{tabular}{lccccccccccccc}
\hline Station no. species & 1 & 2 & 3 & 4 & 5 & 6 & 7 & 8 & 9 & 10 & 11 & 12 \\
\hline 1 G. stupendus & - & & & & & & & & & & \\
2 & G. orinos & 0.22 & - & & & & & & & & & \\
3 G. cf. fossarum & 0.28 & 0.31 & - & & & & & & & & \\
$\mathbf{4}$ G. cf. fossarum & 0.28 & 0.31 & 0.04 & - & & & & & & & \\
5 & G. cf. fossarum & 0.27 & 0.30 & 0.05 & 0.01 & - & & & & & & \\
6 G. gauthieri & 0.68 & 0.73 & 0.76 & 0.76 & 0.75 & - & & & & & \\
7 & G. gauthieri & 0.65 & 0.72 & 0.75 & 0.74 & 0.73 & 0.05 & - & & & & \\
8 G. ibericus & 0.63 & 0.70 & 0.69 & 0.68 & 0.67 & 0.25 & 0.23 & - & & & \\
9 & G. ibericus & 0.61 & 0.69 & 0.64 & 0.60 & 0.59 & 0.51 & 0.49 & 0.32 & - & & \\
10 & G. gauthieri & 0.68 & 0.74 & 0.77 & 0.77 & 0.76 & 0.45 & 0.41 & 0.42 & 0.63 & - & \\
11 & G. gauthieri & 0.68 & 0.75 & 0.78 & 0.78 & 0.77 & 0.44 & 0.41 & 0.42 & 0.63 & 0.06 & - \\
12 G. gauthieri & 0.73 & 0.78 & 0.82 & 0.81 & 0.81 & 0.42 & 0.38 & 0.39 & 0.60 & 0.07 & 0.08 & - \\
\hline
\end{tabular}

\section{TABLE VIII}

Mean intra- and inter-area values of the genetic identity $(I)$ averaged by species, based upon 21 enzyme loci. $G$. orinos and $G$. stupendus have been put together. Inter-areal intra-specific values in bold face; range $I_{\min }-I_{\max }$ in italics.

\begin{tabular}{|c|c|c|c|c|c|c|}
\hline Station no. & $\begin{array}{l}\text { G. orinos } \\
\text { G. stupendus } \\
1,2\end{array}$ & $\begin{array}{l}\text { G. cf. fossarum } \\
3,4,5\end{array}$ & $\begin{array}{l}\text { G. gauthieri } \\
6,7\end{array}$ & $\begin{array}{l}\text { G. ibericus } \\
8\end{array}$ & $\begin{array}{l}\text { G. ibericus } \\
9\end{array}$ & $\begin{array}{l}\text { G. gauthieri } \\
10,11,12\end{array}$ \\
\hline 1,2 & 0.81 & & & & & \\
\hline $3,4,5$ & $\begin{array}{c}0.73 \\
(0.70-0.75)\end{array}$ & $\begin{array}{c}0.98 \\
(0.97-0.99)\end{array}$ & & & & \\
\hline 6,7 & $\begin{array}{c}0.32 \\
(0.27-0.36)\end{array}$ & $\begin{array}{c}0.25 \\
(0.24-0.27)\end{array}$ & 0.96 & & & \\
\hline 8 & $\begin{array}{c}0.34 \\
(0.30-0.38)\end{array}$ & $\begin{array}{c}0.32 \\
(0.31-0.34)\end{array}$ & $\begin{array}{c}0.77 \\
(0.77-0.78)\end{array}$ & - & & \\
\hline 9 & $\begin{array}{c}0.34 \\
(0.30-0.39)\end{array}$ & $\begin{array}{c}0.39 \\
(0.36-0.41)\end{array}$ & $\begin{array}{c}0.52 \\
(0.50-0.53)\end{array}$ & $\begin{array}{l}0.70 \\
-\end{array}$ & - & \\
\hline $10,11,12$ & $\begin{array}{c}0.27 \\
(0.21-0.32)\end{array}$ & $\begin{array}{c}0.21 \\
(0.18-0.23)\end{array}$ & $\begin{array}{c}0.60 \\
(0.56-0.65)\end{array}$ & $\begin{array}{c}0.60 \\
(0.59-0.63)\end{array}$ & $\begin{array}{c}0.38 \\
(0.37-0.41)\end{array}$ & $\begin{array}{c}0.96 \\
(0.95-0.98)\end{array}$ \\
\hline
\end{tabular}

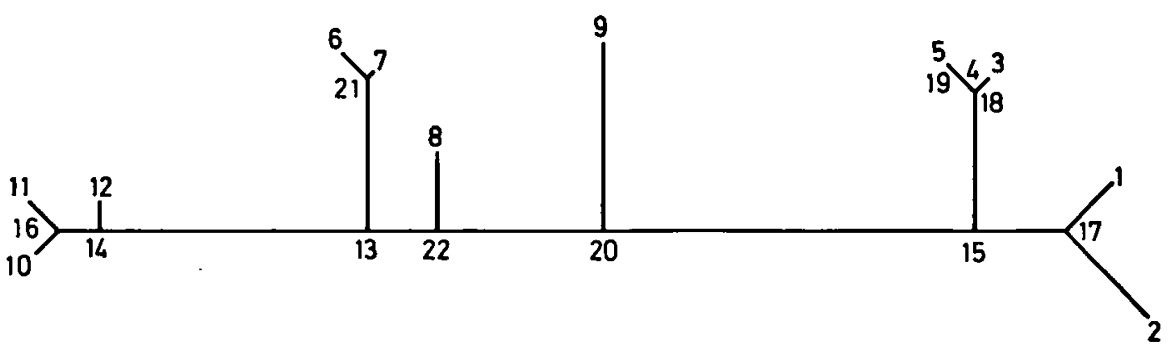

Fig. 5. Jelly "character state" network of 21 loci, based upon Rogers' (1984) HAP algoritm (1-12=OTU 1-12; 13-22 = HTU 13-22). 


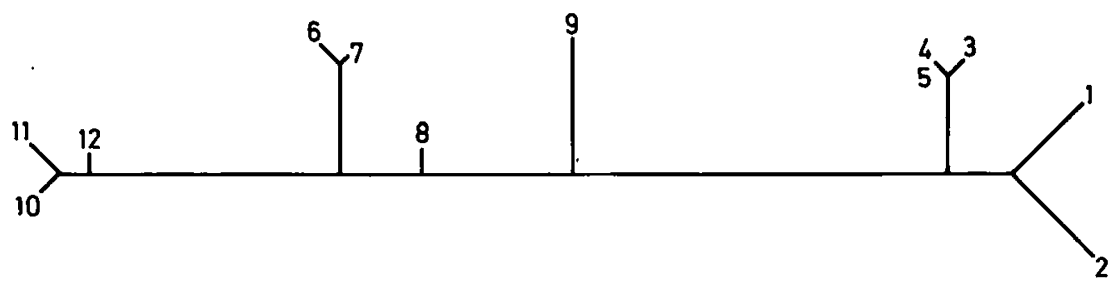

Fig. 6. The distance Wagner dendrogram of fig. 4 represented as an unrooted network.

UPGMA dendrogram (fig. 3) and congruent with the distance Wagner network (figs. 4, 6). Electromorph frequencies of the bifurcation points (hypothetical taxonomic units or HTU's) are given in table IX. Hence, these frequencies allow an estimation of the contribution of each locus to overall changes. "Character state" transformations for the loci studied are indicated in table IX.

\section{Cross-breeding experiments}

The results of the cross-breeding experiments are listed in table X. Experimental conditions were not always optimal. Limitations on the availability of similar age groups resulted in crosses between populations in different phases of their life cycle. Thus, in some cases, old specimens in the last stage of their life had to be

TABLE IX

Electromorph frequencies of hypothetical taxonomic units (HTU's) in the Jelly network (fig. 5) at 20 enzyme loci. HTU's are numbered as in fig. $5 ; \mathrm{a}=$ fastest elecromorph; dominant electromorph(s) in bold face; ${ }^{*}=$ electromorph present, frequency $<0.005$.

\begin{tabular}{|c|c|c|c|c|c|c|c|c|c|c|c|}
\hline \multirow{2}{*}{\multicolumn{2}{|c|}{$\begin{array}{l}\text { Locus } \\
\text { electromorph }\end{array}$}} & \multicolumn{10}{|c|}{ HTU no. } \\
\hline & & 16 & 14 & 13 & 22 & 20 & 15 & 17 & 18 & 19 & 21 \\
\hline Ada-1 & $\begin{array}{l}\mathbf{a} \\
\mathbf{b}\end{array}$ & $\begin{array}{l}0.00 \\
1.00\end{array}$ & $\begin{array}{l}0.00 \\
1.00\end{array}$ & $\begin{array}{l}0.00 \\
1.00\end{array}$ & $\begin{array}{l}0.00 \\
1.00\end{array}$ & $\begin{array}{l}0.00 \\
1.00\end{array}$ & $\begin{array}{l}0.00 \\
1.00\end{array}$ & $\begin{array}{l}0.00 \\
1.00\end{array}$ & $\begin{array}{l}0.00 \\
1.00\end{array}$ & $\begin{array}{l}0.00 \\
1.00\end{array}$ & $\begin{array}{l}0.00 \\
1.00\end{array}$ \\
\hline Alp-1 & $\begin{array}{l}\mathbf{a} \\
\mathbf{b} \\
\mathbf{c}\end{array}$ & $\begin{array}{l}0.00 \\
0.00 \\
1.00\end{array}$ & $\begin{array}{l}0.00 \\
0.00 \\
1.00\end{array}$ & $\begin{array}{l}0.33 \\
0.33 \\
0.33\end{array}$ & $\begin{array}{l}0.00 \\
1.00 \\
0.00\end{array}$ & $\begin{array}{l}0.00 \\
1.00 \\
0.00\end{array}$ & $\begin{array}{l}0.00 \\
1.00 \\
0.00\end{array}$ & $\begin{array}{l}0.00 \\
1.00 \\
0.00\end{array}$ & $\begin{array}{l}0.00 \\
1.00 \\
0.00\end{array}$ & $\begin{array}{l}0.00 \\
1.00 \\
0.00\end{array}$ & $\begin{array}{l}1.00 \\
0.00 \\
0.00\end{array}$ \\
\hline Alp-2 & $\begin{array}{l}\mathbf{a} \\
\mathbf{b}\end{array}$ & $\begin{array}{l}1.00 \\
0.00\end{array}$ & $\begin{array}{l}1.00 \\
0.00\end{array}$ & $\begin{array}{l}1.00 \\
0.00\end{array}$ & $\begin{array}{l}1.00 \\
0.00\end{array}$ & $\begin{array}{l}1.00 \\
0.00\end{array}$ & $\begin{array}{l}1.00 \\
0.00\end{array}$ & $\begin{array}{l}1.00 \\
0.00\end{array}$ & $\begin{array}{l}1.00 \\
0.00\end{array}$ & $\begin{array}{l}1.00 \\
0.00\end{array}$ & $\begin{array}{l}0.70 \\
0.30\end{array}$ \\
\hline$A p k$ & $\begin{array}{l}a \\
b\end{array}$ & $\begin{array}{l}\mathbf{0 . 9 6} \\
0.04\end{array}$ & $\begin{array}{l}\mathbf{0 . 9 7} \\
0.03\end{array}$ & $\begin{array}{l}0.99 \\
0.01\end{array}$ & $\begin{array}{l}0.99 \\
0.01\end{array}$ & $\begin{array}{l}0.99 \\
0.01\end{array}$ & $\begin{array}{l}0.00 \\
1.00\end{array}$ & $\begin{array}{l}0.00 \\
1.00\end{array}$ & $\begin{array}{l}0.00 \\
1.00\end{array}$ & $\begin{array}{l}0.00 \\
1.00\end{array}$ & $\begin{array}{l}1.00 \\
0.00\end{array}$ \\
\hline Est -1 & $\begin{array}{l}a \\
b \\
c\end{array}$ & $\begin{array}{l}1.00 \\
0.00 \\
0.00\end{array}$ & $\begin{array}{l}1.00 \\
0.00 \\
0.00\end{array}$ & $\begin{array}{l}0.00^{*} \\
0.99 \\
0.01\end{array}$ & $\begin{array}{l}0.00^{*} \\
0.99 \\
0.01\end{array}$ & $\begin{array}{l}0.00^{*} \\
0.99 \\
0.01\end{array}$ & $\begin{array}{l}0.00 \\
0.00 \\
1.00\end{array}$ & $\begin{array}{l}0.00 \\
0.00 \\
1.00\end{array}$ & $\begin{array}{l}0.00 \\
0.00 \\
1.00\end{array}$ & $\begin{array}{l}0.00 \\
0.00 \\
1.00\end{array}$ & $\begin{array}{l}0.00 \\
1.00 \\
0.00\end{array}$ \\
\hline Est-2 & $\begin{array}{l}\mathbf{a} \\
\mathbf{b}\end{array}$ & $\begin{array}{l}1.00 \\
0.00\end{array}$ & $\begin{array}{l}1.00 \\
0.00\end{array}$ & $\begin{array}{l}1.00 \\
0.00\end{array}$ & $\begin{array}{l}1.00 \\
0.00\end{array}$ & $\begin{array}{l}0.00 \\
1.00\end{array}$ & $\begin{array}{l}0.00 \\
1.00\end{array}$ & $\begin{array}{l}0.00 \\
1.00\end{array}$ & $\begin{array}{l}0.00 \\
1.00\end{array}$ & $\begin{array}{l}0.00 \\
1.00\end{array}$ & $\begin{array}{l}1.00 \\
0.00\end{array}$ \\
\hline Gdh & $\begin{array}{l}\mathbf{a} \\
\mathbf{b}\end{array}$ & $\begin{array}{l}0.00 \\
1.00\end{array}$ & $\begin{array}{l}0.00 \\
1.00\end{array}$ & $\begin{array}{l}1.00 \\
0.00\end{array}$ & $\begin{array}{l}1.00 \\
0.00\end{array}$ & $\begin{array}{l}1.00 \\
0.00\end{array}$ & $\begin{array}{l}1.00 \\
0.00\end{array}$ & $\begin{array}{l}1.00 \\
0.00\end{array}$ & $\begin{array}{l}1.00 \\
0.00\end{array}$ & $\begin{array}{l}1.00 \\
0.00\end{array}$ & $\begin{array}{l}1.00 \\
0.00\end{array}$ \\
\hline Got-1 & $\begin{array}{l}a \\
b \\
c \\
d\end{array}$ & $\begin{array}{l}0.00 \\
1.00 \\
0.00 \\
0.00\end{array}$ & $\begin{array}{l}0.00 \\
1.00 \\
0.00 \\
0.00\end{array}$ & $\begin{array}{l}0.00 \\
1.00 \\
0.00 \\
0.00\end{array}$ & $\begin{array}{l}0.00 \\
1.00 \\
0.00 \\
0.00\end{array}$ & $\begin{array}{l}1.00 \\
0.00 \\
0.00 \\
0.00\end{array}$ & $\begin{array}{l}1.00 \\
0.00 \\
0.00 \\
0.00\end{array}$ & $\begin{array}{l}0.02 \\
0.00 \\
0.90 \\
0.08\end{array}$ & $\begin{array}{l}1.00 \\
0.00 \\
0.00 \\
0.00\end{array}$ & $\begin{array}{l}1.00 \\
0.00 \\
0.00 \\
0.00\end{array}$ & $\begin{array}{l}0.00 \\
1.00 \\
0.00 \\
0.00\end{array}$ \\
\hline Got-2 & $\begin{array}{l}\mathbf{a} \\
\mathbf{b} \\
\mathbf{c}\end{array}$ & $\begin{array}{l}1.00 \\
0.00 \\
0.00\end{array}$ & $\begin{array}{l}1.00 \\
0.00 \\
0.00\end{array}$ & $\begin{array}{l}1.00 \\
0.00 \\
0.00\end{array}$ & $\begin{array}{l}1.00 \\
0.00 \\
0.00\end{array}$ & $\begin{array}{l}0.60 \\
0.13 \\
0.27\end{array}$ & $\begin{array}{l}0.00 \\
1.00 \\
0.00\end{array}$ & $\begin{array}{l}0.00 \\
1.00 \\
0.00\end{array}$ & $\begin{array}{l}0.00 \\
1.00 \\
0.00\end{array}$ & $\begin{array}{l}0.00 \\
1.00 \\
0.00\end{array}$ & $\begin{array}{l}1.00 \\
0.00 \\
0.00\end{array}$ \\
\hline
\end{tabular}


Table IX (continuation)

\begin{tabular}{|c|c|c|c|c|c|c|c|c|c|c|c|}
\hline \multirow{2}{*}{\multicolumn{2}{|c|}{$\begin{array}{l}\text { Locus } \\
\text { electromorph }\end{array}$}} & \multicolumn{10}{|c|}{ HTU no. } \\
\hline & & 16 & 14 & 13 & 22 & 20 & 15 & 17 & 18 & 19 & 21 \\
\hline \multirow[t]{11}{*}{$G p i$} & $\mathbf{a}$ & $0.00^{*}$ & $0.00^{*}$ & 0.01 & 0.01 & 0.02 & 0.02 & 0.23 & 0.01 & 0.01 & 0.01 \\
\hline & $\mathbf{b}$ & $0.00^{*}$ & $0.00^{*}$ & 0.01 & 0.07 & 0.15 & 0.15 & 0.33 & 0.05 & 0.05 & 0.01 \\
\hline & c & 0.00 & 0.00 & $0.00^{*}$ & $0.00^{*}$ & $0.00^{*}$ & $0.00^{*}$ & 0.02 & $0.00^{*}$ & $0.00^{*}$ & 0.00 \\
\hline & d & 0.05 & 0.05 & 0.13 & 0.26 & 0.13 & 0.13 & 0.07 & 0.04 & 0.04 & 0.13 \\
\hline & e & $0.00^{*}$ & $0.00^{*}$ & $0.00^{*}$ & 0.01 & 0.01 & 0.01 & 0.01 & $0.00^{*}$ & $0.00^{*}$ & $0.00^{*}$ \\
\hline & f & 0.00 & 0.00 & $0.00^{*}$ & 0.02 & 0.03 & 0.03 & 0.02 & 0.14 & 0.14 & $0.00^{*}$ \\
\hline & $\mathbf{g}$ & 0.05 & 0.05 & 0.23 & 0.40 & 0.55 & 0.55 & 0.26 & 0.72 & 0.72 & 0.23 \\
\hline & h & 0.78 & 0.78 & 0.50 & 0.18 & 0.09 & 0.09 & 0.04 & 0.03 & 0.03 & 0.50 \\
\hline & $\mathrm{i}$ & 0.12 & 0.12 & 0.01 & 0.01 & 0.01 & 0.01 & 0.01 & $0.00^{*}$ & $0.00^{*}$ & 0.01 \\
\hline & j & 0.00 & 0.00 & 0.11 & 0.04 & 0.02 & 0.02 & 0.01 & 0.01 & 0.01 & 0.11 \\
\hline & $\mathbf{k}$ & 0.00 & 0.00 & 0.00 & 0.00 & 0.00 & 0.00 & 0.00 & 0.00 & 0.00 & 0.00 \\
\hline \multirow[t]{3}{*}{$H k-1$} & $\mathbf{a}$ & 0.00 & 0.00 & 0.00 & 0.00 & 0.33 & 0.62 & 0.62 & 1.00 & 1.00 & 0.00 \\
\hline & b & 1.00 & 1.00 & 1.00 & 1.00 & 0.40 & 0.28 & 0.28 & 0.00 & 0.00 & 1.00 \\
\hline & c & 0.00 & 0.00 & 0.00 & 0.00 & 0.27 & 0.10 & 0.10 & 0.00 & 0.00 & 0.00 \\
\hline \multirow[t]{5}{*}{$M d h-1$} & $\mathbf{a}$ & 0.00 & 0.00 & 0.02 & 0.05 & 0.05 & 0.05 & 0.90 & 0.00 & 0.00 & 0.00 \\
\hline & b & 0.00 & 0.00 & 0.33 & 0.87 & 0.87 & 0.87 & 0.10 & 1.00 & 1.00 & 0.00 \\
\hline & c & 0.00 & 0.00 & 0.33 & 0.05 & 0.05 & 0.05 & 0.00 & 0.00 & 0.00 & 1.00 \\
\hline & d & 0.98 & 0.98 & 0.32 & 0.03 & 0.03 & 0.03 & 0.00 & 0.00 & 0.00 & 0.00 \\
\hline & e & 0.02 & 0.02 & $0.00^{*}$ & $0.00^{*}$ & $0.00^{*}$ & 0.00 & 0.00 & 0.00 & 0.00 & 0.00 \\
\hline \multirow[t]{2}{*}{$M d h-2$} & $\mathbf{a}$ & 0.00 & 0.00 & 0.00 & 0.00 & 0.00 & 0.00 & 0.00 & 0.48 & 0.64 & 0.00 \\
\hline & b & 1.00 & 1.00 & 1.00 & 1.00 & 1.00 & 1.00 & 1.00 & 0.52 & 0.36 & 1.00 \\
\hline \multirow[t]{3}{*}{$M e$} & $a$ & 0.00 & 0.00 & 0.32 & 0.60 & 0.60 & 0.00 & 0.00 & 0.00 & 0.00 & 0.00 \\
\hline & b & 0.00 & 0.00 & 0.45 & 0.34 & 0.34 & 1.00 & 1.00 & 1.00 & 1.00 & 1.00 \\
\hline & c & 1.00 & 1.00 & 0.23 & 0.06 & 0.06 & 0.00 & 0.00 & 0.00 & 0.00 & 0.00 \\
\hline \multirow[t]{7}{*}{$M p i$} & $\mathbf{a}$ & 0.00 & 0.02 & 0.23 & 0.13 & 0.03 & 0.01 & 0.01 & $0.00^{*}$ & 0.00 & 1.00 \\
\hline & b & 0.00 & 0.01 & 0.16 & 0.25 & 0.10 & 0.05 & 0.05 & 0.01 & 0.00 & 0.00 \\
\hline & c & 0.02 & 0.03 & 0.17 & 0.27 & 0.50 & 0.48 & 0.48 & 0.13 & 0.00 & 0.00 \\
\hline & d & 0.00 & $0.00^{*}$ & 0.02 & 0.04 & 0.08 & 0.10 & 0.10 & 0.03 & 0.00 & 0.00 \\
\hline & e & 0.00 & $0.00^{*}$ & 0.06 & 0.09 & 0.18 & 0.26 & 0.26 & 0.56 & 1.00 & 0.00 \\
\hline & $f$ & 0.30 & 0.35 & 0.15 & 0.10 & 0.08 & 0.09 & 0.09 & 0.26 & 0.00 & 0.00 \\
\hline & $\mathbf{g}$ & 0.68 & 0.59 & 0.21 & 0.12 & 0.03 & 0.01 & 0.01 & $0.00^{*}$ & 0.00 & 0.00 \\
\hline \multirow[t]{6}{*}{ Pep-2 } & $\mathbf{a}$ & 0.02 & 0.02 & 0.10 & 0.10 & 0.34 & 0.14 & 0.14 & 0.00 & 0.00 & 0.00 \\
\hline & $\mathbf{b}$ & 0.13 & 0.13 & 0.59 & 0.59 & 0.26 & 0.06 & 0.06 & 0.00 & 0.00 & 1.00 \\
\hline & c & 0.06 & 0.06 & 0.00 & 0.00 & 0.00 & 0.00 & 0.00 & 0.00 & 0.00 & 0.00 \\
\hline & d & 0.78 & 0.78 & 0.24 & 0.24 & 0.11 & 0.02 & 0.02 & 0.00 & 0.00 & 0.00 \\
\hline & e & 0.01 & 0.01 & 0.06 & 0.06 & 0.29 & 0.76 & 0.76 & 1.00 & 1.00 & 0.00 \\
\hline & f & $0.00^{*}$ & $0.00^{*}$ & 0.01 & 0.01 & 0.00 & 0.02 & 0.02 & 0.00 & 0.00 & 0.00 \\
\hline \multirow[t]{2}{*}{ Pep-3 } & $\mathbf{a}$ & 1.00 & 1.00 & 1.00 & 1.00 & 1.00 & 0.00 & 0.00 & 0.00 & 0.00 & 1.00 \\
\hline & b & 0.00 & 0.00 & 1.00 & 0.00 & 1.00 & 1.00 & 1.00 & 1.00 & 1.00 & 0.00 \\
\hline \multirow[t]{5}{*}{ Pep-4 } & $\mathbf{a}$ & 0.90 & 0.90 & 0.99 & 0.99 & 0.99 & 0.69 & 0.69 & 0.00 & 0.00 & 1.00 \\
\hline & b & 0.00 & 0.00 & 0.00 & 0.00 & 0.00 & 0.00 & 0.00 & 0.00 & 0.00 & 0.00 \\
\hline & c & 0.09 & 0.09 & $0.00^{*}$ & $0.00^{*}$ & $0.00^{*}$ & $0.00^{*}$ & $0.00^{*}$ & 0.00 & 0.00 & 0.00 \\
\hline & d & $0.00^{*}$ & $0.00^{*}$ & $0.00^{*}$ & $0.00^{*}$ & $0.00^{*}$ & 0.08 & 0.08 & 0.00 & 0.00 & 0.00 \\
\hline & $\mathbf{e}$ & 0.01 & 0.01 & 0.01 & 0.01 & 0.01 & 0.23 & 0.23 & 1.00 & 1.00 & 0.00 \\
\hline \multirow[t]{2}{*}{ 6-Pgd } & $\mathbf{a}$ & 0.00 & 0.00 & 0.00 & 0.00 & 0.00 & 1.00 & 1.00 & 1.00 & 1.00 & 0.00 \\
\hline & $\mathbf{b}$ & 1.00 & 1.00 & 1.00 & 1.00 & 1.00 & 0.00 & 0.00 & 0.00 & 1.00 & 1.00 \\
\hline \multirow[t]{2}{*}{$P k$} & $\mathbf{a}$ & 0.00 & 0.81 & 1.00 & 1.00 & 1.00 & 0.00 & 0.00 & 0.00 & 0.00 & 1.00 \\
\hline & b & 1.00 & 0.19 & 0.00 & 0.00 & 0.00 & 1.00 & 1.00 & 1.00 & 1.00 & 0.00 \\
\hline
\end{tabular}


TABLE X

Results of cross-breeding experiments between populations of $G$. gauthieri and $G$. ibericus: $g_{7}=G$. gauthieri from station $7 ; g_{10}=G$. gauthieri from station $10 ; i_{8}=G$. ibericus from station $8 ; i_{9}=G$. ibericus from station 9 .

\begin{tabular}{|c|c|c|c|c|c|}
\hline $\begin{array}{c}\text { Cross } \\
\wp \quad 0\end{array}$ & $\mathrm{~N}$ of $0^{\circ} \mathrm{O}^{\circ}$ & $\mathrm{N}$ of $\$ Q$ & $\begin{array}{c}\mathbf{N} \text { of } \\
\text { ovigerous } ९ ९\end{array}$ & $\begin{array}{l}N \text { of ovigerous } \\
९ Q \text { with offspring }\end{array}$ & $\begin{array}{c}\text { F2 } \\
\text { obtained }\end{array}$ \\
\hline$g_{7} \times g_{7}$ & 50 & 50 & 23 & 5 & + \\
\hline$g_{10} \times g_{10}$ & 75 & 54 & 24 & 7 & + \\
\hline$i_{8} \times i_{8}$ & 53 & 45 & 31 & 9 & + \\
\hline$i_{g} \times i_{g}$ & 100 & 100 & 69 & 27 & + \\
\hline$g_{10} \times g_{7}$ & 94 & 93 & 33 & 11 & - \\
\hline$g_{7} \times g_{10}$ & 39 & 26 & 12 & 7 & + \\
\hline$i_{8} \times g_{7}$ & 50 & 50 & 41 & - & - \\
\hline$g_{7} \times i_{8}$ & 76 & 61 & 30 & 9 & + \\
\hline$i_{9} \times i_{8}$ & 50 & 16 & 40 & 8 & + \\
\hline$i_{8} \times i_{g}$ & 78 & 78 & 69 & 27 & + \\
\hline
\end{tabular}

crossed with young almost immature specimens from other populations.

All crosses but one gave $F_{1}$ offspring. Cross $i_{8} \times g_{7}$ (females $G$. ibericus from station 8 and males $G$. gauthieri from station 7) failed to yield offspring. Except for the reference crosses, $F_{2}$ in very low numbers were obtained from crosses $g_{7} \times g_{10}$ (females G. gauthieri from station 7 and males $G$. gauthieri from station 10), $i g \times i_{8}$ (females $G$. ibericus from station 9 and males $G$. ibericus from station 8$), i_{8} \times i_{g}$ (females $G$. ibericus from station 8 and males $G$. ibericus from station 9) and $g_{7} \times i_{8}$ (females $G$. gauthieri from station 7 and males $G$. ibericus from station 8).

\section{DISCUSSION AND CONCLUSIONS}

\section{Genetic diversity}

Mean $I$-values describing various taxonomic levels are given by several authors (Ayala et al., 1973; Avise \& Smith, 1977; Ferguson, 1980; Thorpe, 1982; Menken \& Ulenberg, 1987; see table XI). Inter-areal values of $I$ between French and Iberian populations separated by the Pyrenean Mountain chain are remarkably low $(I=0.18-0.41$, mean: $0.28 ; D=0.88-1.75$, mean: 1.30). Referring to table XI, these values locate at the generic level. Similar results were reported by Siegismund et al. (1985) and Skadsheim \& Siegismund (1986) who stated that " within the genus Gammarus, genetic identities in species pairs are as low as those between Gammarus spp. and species in other genera".

Intra-specific values of $I$ among $G$. gauthieri populations from the Valladolid area and Cuenca area $(I=0.56-0.65 ; D=0.57-0.44)$, and $G$. ibericus populations from stations 8 and $9(I=0.70 ; D=0.36)$ are low for conspecific populations. The Valladolid area and the Serranía de Cuenca are separated by the Sierra de Gredos and the Sierra de Guadarrama. Stations 8 and 9, although geographically much closer, belong (see table I) to the Atlantic Ocean and Mediterranean drainage systems, respectively, separated by the Serranía de Cuenca. These mountain chains have also been recognized by Lop (1987) as barriers preventing genetic exchange. According to table XI, these values are below the expected subspecies level and range about what is commonly attributed to sibling species, and, to a lesser extent, congeneric species.

Intra-specific values of $D$ in $G$. gauthieri range from 0.02-0.57 ( $I=0.56-0.98)$. Lop (1987), in his study of the Echinogammarus berilloni group from the Iberian Peninsula, found intra-specific values of $D$ ranging from 0.05-0.26 ( $I=0.77$ $0.95)$. Although these values diverge less than 
TABLE XI

Values of genetic identity on various taxonomic levels based upon literature data: 1 = Ayala et al. (1973); $2=$ Avise \& Smith (1977); 3 = Thorpe (1982); 4 = Ferguson (1980); 5 = Menken \& Ulenberg (1987); $I=$ genetic identity (Nei, 1972); S.E. = standard error.

\begin{tabular}{|c|c|c|c|c|c|}
\hline Taxonomic level & $\begin{array}{l}1 \\
I\end{array}$ & S.E. & $\begin{array}{l}3 \\
I\end{array}$ & $\begin{array}{l}4 \\
I\end{array}$ & $\begin{array}{l}5 \\
\text { Range of } I\end{array}$ \\
\hline Populations & $0.970 \pm 0.006$ & $0.980 \pm 0.004$ & $0.960 \pm 0.032$ & - & $0.78-1.00$ \\
\hline Subspecies & $0.795 \pm 0.013$ & $0.843 \pm 0.013$ & - & - & $0.80-0.98$ \\
\hline Semispecies & $0.873 \pm 0.012$ & - & - & 一 & - \\
\hline Sibling species & $0.517 \pm 0.024$ & - & - & 0.626 & $0.00-0.98$ \\
\hline Species & $0.352 \pm 0.0023$ & $0.544 \pm 0.020$ & $0.540 \pm 0.168$ & 0.567 & $0.00-0.90$ \\
\hline Genera & - & $0.294 \pm 0.020$ & $0.273 \pm 0.107$ & 0.362 & $0.00-0.60$ \\
\hline
\end{tabular}

in G. gauthieri, it can be concluded that gammarids from the Iberian Peninsula tend to be genetically much more differentiated at the species level than species groups reported in the literature, or even Gammarus spp. in France: the $I$-value for $G$. orinos and $G$. stupendus is 0.80 $(D=0.22)$ and other data suggest that lower values are not likely to be found (Scheepmaker, in prep.). There is a discrepancy between morphological and allozymic differentiation. Evolution of morphological characters may not be constant. If particular morphological types tend to be conserved, the relation between morphological and genetic divergence will decrease.

Morphologically, $G$. cf. fossarum is related to $G$. orinos rather than to $G$. fossarum Koch. From this study it may appear that $G$. cf. fossarum should be considered as a separate species. Further experiments showed that $G$. cf. fossarum from the Pyrenees and $G$. fossarum are not conspecific (Scheepmaker, in prep.). In these experiments however, some populations of $G$. cf. fossarum and $G$. orinos showed a considerable overlap in genetic variation. Thus, the taxonomic status of $G$. cf. fossarum remains provisionally unsolved and will be treated in a future study.

\section{Genetic divergence and evolutionary rates}

Several authors have attempted to explain differences in genetic variation of enzymes within and among populations and species (e.g. metabolic function: Johnson, 1976; Selander, 1976; for Crustacea, Hedgecock et al., 1982; molecular structure and subunit size: Koehn \& Eanes, 1978). Evidence for certain loci being more conservative than others has been discussed by Avise (1975), Sarich (1977), Thorpe (1982) and Menken \& Ulenberg (1987). Sarich (1977) distinguished "fast" and "slow" evolving loci. According to Menken \& Ulenberg (1987), the ability to differentiate among the investigated species may depend on different evolutionary rates. Striking is, in this study, that a number of loci appear to be fixed for specific electromorphs in many samples in which the alternative allelic complement is rare, or absent (this was also found in other studies on amphipods, e.g. Siegismund et al., 1985; Bulnheim, 1985; Bulnheim \& Scholl, 1981, 1986; Scheepmaker, unpubl.). This may be consequent to a local polymorphism fixed for the mutant electromorph by drift or directional selection. In either case, it may be justified to calibrate the molecular clock for a given set of loci in a group of closely related species, as long as the evolutionary rates for the individual loci remain constant.

These "fixed" loci are invariable or almost invariable across the populations of a species or species group over long distances. For instance, the Lap locus is invariable for all members studied of the $G$. pulex group (sensu Karaman \& Pinkster, 1977) ranging from Holland to central Spain. Within the same group, the 6-Pgd 
and $P e p-3$ loci are fixed for one electromorph in all members from the Iberian Peninsula (and thus diagnostic with respect to those north of the Pyrenees). All the other members, from the Pyrenees north up to Holland, are fixed invariably for the same alternative electromorph. At the species level, electromorphs are sometimes invariable over long distances across most members, but diagnostic for one or two of them (e.g. G. fossarum, G. orinos, G. cf. fossarum and $G$. stupendus versus $G$. wautieri and $G$. pulex at the $P k$ locus; Scheepmaker, in prep.). Occasionally, this was also observed at the intra-specific level (viz. $A d a-1$ and $H k-1^{c}$ at station $9 ; P k$ at station 12).

In the present study, such loci seem to be represented by $A p k, A d a-1, A l p-1, A l p-2$, Est-1, Est2, Gdh, Hk-1, Lap, Pep-3, 6-Pgd, and Pk. Alternatively, at both inter- en intra-specific level, loci such as Gpi, Mpi, Pep-2, Pep-4, Got-1, Got-2, $M d h-1$, and $M d h-2$ may be highly variable. The remaining loci may range somewhere in between. This bimodality in levels of polymorphism (and possibly in rates of evolutionary change) has been reported by several authors (reviewed by Thorpe, 1982 and Menken \& Ulenberg, 1987).

When calculating distance matrices (and the resulting dendrograms) no distinction is made between slow and fast evolving loci. However, since the Wagner and UPGMA dendrograms and the Jelly network are similar in overall view (figs. 3, 4,6), major splits and path-lengths can be brought back to "character state" changes in the Jelly network (fig. 4, table IX). Employing the Jelly computer program, an estimate of the amount of change and the proportion of fast and slow loci involved can be made.

Major splits in the Jelly network are generated by loci that either exhibit a low level of polymorphism, or are monomorphic (e.g. Apk, Alp-1, Alp-2, Est-1, Est-2, Gdh, Hk-1, 6 -Pgd, $P k$; see table IX). The contribution of highly variable loci such as $G p i, M p i, P e p-2$, and $P e p-4$ is reduced. Alternatively, these loci (reflecting local variation in conspecific populations) determine largely the topology of the network at the OTU level (e.g. G. cf. fossarum from stations 3, 4, 5; G. gauthieri from stations 6 and 7 , and stations $10,11,12$ ).

\section{Cross-breeding experiments}

In cross-breeding experiments, French and Iberian species have proved to be completely infertile among each other (e.g. G. ibericus vs. G. orinos, Pinkster \& Scholl, 1984; G. gauthieri from Spain vs. G. wautieri and $G$. fossarum, Goedmakers \& Roux, 1975). However, the results of the cross-breeding experiments in this study (table X) do not contribute to the resolution of the phylogenetic relationships or the taxonomic status of genetically well differentiated, but morphologically similar allopatric forms of $G$. gauthieri and $G$. ibericus from the Iberian Peninsula.

Despite of the considerable amount of genetic divergence between allopatric populations of both $G$. gauthieri and $G$. ibericus, complete reproductive isolation has not yet been achieved. However, it can also be concluded that these conspecific allopatric populations are not completely interfertile. Another remarkable result is provided by cross $g_{7} \times i_{8}$ (females $G$. gauthieri from station 7 and males $G$. ibericus from station 8), yielding F2 offspring. These populations are both genetically and morphologically clearly distinct.

It could be argued that morphologically identical and only partially reproductively isolated forms should be considered conspecific. However, in the case of genetically and morphologically well-differentiated forms such as G. gauthieri from station 7 and G. ibericus from station 8 , which still prove to be at least partially interfertile, reproductive isolation as criterion in taxonomy becomes heavily undermined.

Furthermore, it is interesting that both morphologically and genetically much less differentiated species in France are often reproductively isolated completely (e.g. G. pulex vs. G. fossarum, Wautier \& Roux, 1959; Roux, 1971a; $G$. wautieri vs. G. pulex and G. fossarum, Roux, $1971 \mathrm{a} ; G$. stupendus vs. G. orinos and $G$. fossarum, Pinkster, 1983; Scheepmaker, 1987; G. orinos 
vs. G. cf. fossarum and G. fossarum, Scheepmaker, in prep.). For the present study, crossbreeding experiments were also performed with $G$. cf. fossarum from station 3 (fig. 1B) and $G$. ibericus from station 9 showing electromorphs that characterize the French forms. The technical difficulties mentioned above, and increased mortality in one of the populations involved, caused these experiments to be unsuccessful.

Roux (1971b) demonstrated that sympatric occurrence enhances reproductive isolation. This suggests that reproductive isolation may not be related to the degree of genetic differentiation, but rather to the mode of speciation (e.g. allopatric vs. sympatric).

\section{Genetic distance and divergence time}

Nei's $D$ claims to be a measure for genetic divergence time (Nei, 1975). However, it is obvious that the divergence time thus obtained is strongly dependent on the number and the proportions of fast and slow loci involved and the organisms studied. Moreover, the same loci appear to contribute to a different extent to $D$ values in comparable stages of evolutionary divergence depending on the taxonomic group studied (De Matthaeis et al., 1983). These reasons might explain in part the discordance of approximations of $D$ that have been made in the past: proposed values of one unit $D$ range from 5 to $20 \mathrm{mY}$ (Thorpe, 1982; Sbordoni et al., 1980; Bert, 1986).

Sbordoni (1982), who reviewed speciation in cave animals, concluded that troglobytes, being confined to caves and not having the opportunity to disperse over long distances, are excellent paleogeographic monitors and their present distribution generally reflects particular paleogeographic events which have been dated by geologists. Several authors (Sbordoni et al., 1980; Caccone et al., 1982; De Matthaeis et al., 1982; Cobolli Sbordoni et al., 1987; Caccone et al., 1986) studied divergence time in cavedwelling organisms in the Mediterranean region. These authors reported a close agreement of paleogeographic and paleoclima- tological evidence and divergence time according to Nei (1975) (viz. a one unit $D$ value of $5 \mathrm{mY}$ ). Thus, from these studies, with in most cases related groups of organisms, it appears that Nei's (1975) estimate of divergence time provides a reasonable approximation. Therefore, to check if there is any agreement between ranges of divergence time and paleogeographical and paleoclimatological evidence, we calculated divergence time according to Nei (1975).

Two alternative hypotheses can be proposed to account for the present-day divergence of French and Iberian members of the G. pulex group. According to the first, French populations of $G$. orinos, $G$. stupendus and $G$. cf. fossarum and Iberian populations of $G$. gauthieri and $G$. ibericus may have originated from the same stock and separated in the past by some barrier. Evidence for the Pyrenean mountain chain as a barrier preventing gene flow is provided by high $D$-values between species from France and the Iberian Peninsula. However, calculated divergence time should be in the same order as geological data on the uplifting of the Pyrenees. The Alpine orogenesis in the Pyrenean belt started in the Late Cretaceous. About that period the Iberian Peninsula was intermittently separated from the European continent by marine transgressions, and during the Santonian, the Alpine sea was connected with the Atlantic (Plaziat, 1981). Although folding first occurred at the end of the Cretaceous, major folding occurred at the end of the Oligocene and vertical movements resulting into the present-day relief may be largely of Pleistocene age, thus less than $2 \mathrm{mY}$ ago (Mattauer \& Henry, 1974). It is not clear in which period the Pyrenees became a barrier to gene flow. Minimum and maximum $D$-values according to Nei (1975) among the Spanish and French populations studied range from 8.7 to $4.4 \mathrm{mY}$ (Upper Miocene-Middle Pliocene; mean 6.5 $\mathrm{mY})$.

If the Pyrenees formed a barrier to gene flow, the fall in sea level during the Messinian salinity crisis (Hsü, 1972; Hsü et al., 1977; Rögl \& Steininger, 1983) possibly provided a 
temporary migration pathway along the emerging coastal plains bordering the eastern boundaries of southern France and Spain; this may also have happened during the Pleistocene glaciations and the subsequent falls in sea level. Supporting evidence may be provided by the "French" electromorphs Est-2b, Got-1a and Got $-2^{c}$ in G. ibericus from station 9. Moreover, the presence of this electrophoretically distinct form coincides with the southernmost distribution limit in this area of the western European Echinogammarus berilloni group (Lop, 1987). During the Messinian, G. gauthieri was probably able to disperse to North Africa.

Although divergence data and geological evidence do not conflict, they are hardly informative and leave the way open to a second hypothesis. According to this hypothesis, both French and Iberian populations originated from the same stock, probably in the Balkan and Asia Minor, areas considered as "centre of origin" of the G. pulex group because of its species richness (Karaman \& Pinkster, 1987). According to this theory, (ancestors of) West European species (viz. the French species) moved along the northern side of the Mediterranean to the west, whereas the Iberian species originated from ancestors that migrated along the North African side of the Mediterranean after the junction of the Eurasian and African plates in the Middle Miocene (about $10 \mathrm{mY}$ ago). During the Messinian salinity crisis (6-5 $\mathrm{mY}$ ago) thèse forms where able to migrate from North Africa to Spain. Supporting evidence for this theory is provided by the distribution areas of $G$. gauthieri and the Echinogammarus simoni group, which both have their largest extension in North Africa (Karaman \& Pinkster, 1977).

The hypothesis proposing that the ancestors of $G$. gauthieri and $G$. ibericus originated from Europe and not from Africa also agrees with the "paleo-ecological principle" proposed by Golikov \& Tzvetkova (1972). Because of the improbability of a major adaptive shift of a species group as a whole to different ecological conditions, this principle presumes that the major part of the contemporary distribution area of a species group is still limited primarily to ecological conditions that are similar to those in which it originated. For gammarids, these particular conditions are found in the more temperate regions. During the Middle Miocene, perhaps except at higher altitudes, temperate conditions were not yet prevailing in North Africa. Hence, according to this hypothesis, the extension of gammarids is most likely to represent an adaptive shift of some species to the more extreme conditions in a remote area rather than evidence for a presumed alternative centre of origin of the species group.

As a third alternative, a combination of both dispersion routes may account in part for the present-day distribution.

\section{Paleoclimatological aspects of inter-areal genetic divergence}

A general trend towards a cooler and dryer climate occurred during the Late Miocene (Maldonado, 1985; Hsü et al., 1977), while during the Pliocene a sequence of cool and cold phases started (Van der Hammen et al., 1971; Lop, 1987). This sequence preceded the Pleistocene glaciations. During the cold phases, major shifts in vegetation zones occurred. North of the Pyrenees boreal conditions may have prevailed whereas the Iberian Peninsula was characterized by a steppe and desert climate (Van der Hammen et al., 1971). In the Iberian Peninsula the semi-permanent desiccation of riverbeds and watercourses as a consequence of the dry climate may have resulted in long-term isolation of populations and consequently in increased levels of genetic divergence. This has also been suggested by Lop (1987) who found high levels of intraspecific divergence in Echinogammarus spp. from the Iberian Peninsula.

Alternatively, under the more humid boreal conditions north of the Pyrenees, the opportunity to disperse over long distances was maintained. This hypothesis appears to be supported by $D$-values among the Spanish and French populations studied. The mean $D$-value among 
Spanish populations is 0.47 with a maximum of 1.00 , which corresponds to about 2.3 and 5 $\mathrm{mY}$, respectively, and suggests a divergence of Pliocene age. The mean $D$-value of French populations is 0.22 (maximum 0.35 ), corresponding to 1.1 and $1.7 \mathrm{mY}$, respectively, suggesting a divergence of Pleistocene age. In this period, cold phases were likely to be responsible for an increased isolation among populations north of the Pyrenees.

It may be concluded that the orders of divergence resolved and data on paleogeographical and paleoclimatological evidence do not conflict. These data provide an explanation for differences in inter-areal genetic divergence of French and Iberian populations. However, they do neither contribute to the solution of dispersion routes nor to the appreciation of differences in the evolutionary rates of the loci involved. Electrophoretic screening of North African populations of $G$. gauthieri seems indispensable to resolve the former problem, and to potentially provide an initial calibration of the molecular clock for the $G$. pulex group for the given set of loci.

\section{ACKNOWLEDGEMENTS}

We wish to thank Dr. C. B. Crabtree, Dr. S. B. J. Menken, Dr. H. R. Siegismund and Ir. J. Notenboom for commenting on the manuscript; Dr. W. N. Ellis for introducing us in his computer program Jelly; Dr. T. A. Wijmstra for valuable suggestions concerning the paleoclimatology of the Iberian Peninsula; and Mr. B. van den Hoek for his help in running the cross-breeding experiments.

\section{REFERENCES}

Avise, J. C., 1975. Systematic value of electrophoretic data. Syst. Zool., 23: 465-481.

Avise, J. C. \&. H. SMrth, 1977. Gene frequency comparisons between sunfish (Centrarchidae) populations at various stages of evolutionary divergence. Syst. Zool., 26: 319-335.

Ayala, F. J. J. R. Powell, 1972. Allozymes as diagnostic characters of sibling species of Drosophila. Proc. natn. Acad. Sci. U.S.A., 69 (5): 1094-1096.

Ayala, F. J., J. R. Powell, M. L. Tracey, C. A. Mourão \&. Pérez-Salas, 1972. Enzyme variability in the Drosophila willistoni group, IV.
Genic variation in natural populations of Drosophila willistoni. Genetics, 70: 113-139.

Ayala, F. J., M. L. Tracey, D. Hedgecock \& R. C. Richmond, 1973. Genetic differentiation during the speciation process in Drosophila. Evolution, 28: 576-592.

Bert, T. M., 1986. Speciation in western Atlantic stone crabs (genus Menippe): the role of geological processes and climatic events in the formation and distribution of species. Mar. Biol., 93: 157-170.

Brewer, G. J., 1970. An introduction to isozyme techniques: 1-186 (Academic Press, London).

Bulnheim, H. P., 1985. Genetic differentiation between natural populations of Gammarus tigrinus (Crustacea, Amphipoda) with reference to its range extension in European continental waters. Arch. Hydrobiol., 102: 273-290.

Bulnheim, H. P. \& A. Scholl, 1981. Electrophoretic approach to the biochemical systematics of gammarids. Helgoländ. wiss. Meeresunters., 34: 391-400.

- \& - - 1986. Genetic differentiation between populations of Talitrus saltator and Talorchestia deshayesii (Crustacea, Amphipoda) from coastal areas of the north-western European continent. Mar. Biol., 92: 525-536.

Buth, D. G., 1984. The application of electrophoretic data in systematic studies. Ann. Rev. Ecol. Syst., 15: 501-522.

Caccone, A., G. Allegrucci, D. Cesaroni, M. Cobolli Sbordoni, E. de Matthaeis, G. la Rosa \& V. SBordonI, 1986. Genetic variability and divergence between cave dwelling populations of Typhlocirolana from Majorca and Sicily. Biochemical Systematics and Ecology, 14 (2): 215-221.

Caccone, A., M. Cobolli Sbordoni, E. de Matthaeis \& V. Sbordoni, 1982. Una datazione su base genetico-moleculare della divergenza tra specie cavernicole e marine di Sferomidi (gen. Monolistra e Sphaeroma, Crustacea, Isopoda). Lavori Soc. ital. Biogeogr., (N.S.) 7: 853-867.

Glayton, J. W. D. N. Tretiak, 1972. Amine-citrate buffers for $\mathrm{pH}$ control in starch gel electrophoresis. J. Fish. Res. Bd. Canada, 29: 1169-1172.

Cobolli Sbordoni, M., E. de Matthaeis, G. la Rosa, M. Mattoccia \& A. Vigna Taglianti, 1987. Biochemical differentiation and divergence time in the Canarian genus Eutrichopus (Coleoptera, Carabidae). Preprint of the International Symposium on Biogeographical Aspects of Insularity, Rome, 18-22 May 1987.

Dercourt, J., L. P. Zonenshain, L. E. Ricou, V. G. Kazmin, X. Le Pichon, A. L. Knipper, C. GrandJacquet, I. M. Sborshchikov, J. Boulin, O. Sorokhtin, J. Geyssant, C. Lepvrier, B. BijuDuval, J. C. Sibuet, L. A. Savostin, M. Westryal. * J. P. Lauer, 1985. Présentation de 9 cartes paléogéographiques au $1 / 2.000 .000$ s'étendant de 
l'Atlantique au Pamir pour la période du Lias à l'actuel. Bull. Soc. geol. France, (8) 1 (5): 637-652; 10 cartes en couleur hors texte (Atlas).

Ellis, W. N., 1987. Jelly version 1.06; a program for the Macintosh computer for the generation of Wagner character state networks using allele frequency characters (available from the author, c/o Institute of Taxonomic Zoology, Entomology Department, Plantage Middenlaan 64, 1018 DH Amsterdam).

Farris, J. S., 1972. Estimating phylogenetic trees from distance matrices. Am. Nat., 106: 645-668.

,-- 1981 . Distance data in phylogenetic analysis. In: V. A. Brooks D. R. Brooks eds., Advances in cladistics: Proceedings of the first meeting of the Willi Hennig Society: 3-23 (New York Botanical Garden, Bronx).

Ferguson, A., 1980. Biochemical systematics and evolution: 1-194 (Blackie, Glasgow/London).

García de Jalón Lastra, D. \& M. González del Tánago, 1986. Metodos biologicos para el estudio de la calidad de las aguas. Aplicación a la cuenca del Duero. Icona Monografias, 45: 1-244.

Giblett, E. R., 1969. Genetic markers in human blood: 1-629 (Blackwell, Oxford).

Goedmakers, A., 1974. Les Gammaridae (CrustacésAmphipodes) du Massif Central. Bull. zoöl. Mus. Univ. Amsterdam, 3 (23): 211-220.

Goedmakers, A. \& A. L. Roux, 1975. Essais d'hybridation entre plusieurs populations du groupe pulex (Crustacés, Amphipodes). Crustaceana, 24 (1): 99-109.

Golikov, A. N. \& N. L. Tzvetkova, 1972. The ecological principle of evolutionary reconstruction as illustrated by marine animals. Mar. Biol., 14 (1): 1-9.

Hammen, T. van der, T. A. Wijmstra \& W. H. Zagwijn, 1971. The floral record of the Late Cenozoic of Europe. In: K. K. Turekian ed., The Late Cenozoic ages: $391-424$ (Yale University Press, New Haven \& London).

Harris, H. \&. A. Hopkinson, 1976. Handbook of enzyme electrophoresis in human genetics: 1-306 (North Holland, Amsterdam).

Hedgecock, D., M. L. Tracey \& M. L. Nelson, 1982. Genetics. In: E. Buiss ed., The biology of Crustacea: 283-403 (Academic Press, New York).

Hsü, K. J., 1972. When the Mediterranean dried up. Scient. Am., 227 (6): 26-36.

Hsü, K. J., L. Montadert, D. Bernouilei, M. B. Cita, A. Erickson, R. E. Garrison, R. B. Kidd, F. Mèlierés, C. Müller \& R. Wright, 1977. History of the Mediterranean salinity crisis. Nature, 267: 399-403.

JeLNES, J. E., 1971. Identification of hexokinase and localisation of a fructokinase and tetrazolium oxidase locus in Drosophila melanogaster. Hereditas, 67: 291-293.

Johnson, G. B., 1976. Genetic polymorphism and enzyme function. In: F. J. Ayala ed., Molecular evolution: 46-59 (Sinauer, Sunderland, Mass.).
Karaman, G. S. \& S. Pinkster, 1977. Freshwater Gammarus species from Europe, North Africa and adjacent regions of Asia (Crustacea-Amphipoda). Part 1. Gammarus pulex-group and related species. Bijdr. Dierk., 47 (1): 1-97.

- — \& - 1987. Freshwater Gammarus species from Europe, North Africa and adjacent regions of Asia (Crustacea-Amphipoda). Part III. Gammarus balcanicus-group and related species. Bijdr. Dierk., 57 (2): 207-260.

Kоенn, R. K. \& W. F. EAnes, 1978. Molecular structure and protein variation within and among populations. Evolut. Biol., 11: 39-100.

Lop, A. F., 1987. Análisis de la variabilidad intra e interspecífica en el grupo de Echinogrammarus berilloni (Crustacea, Amphipoda): [i-xvi], 1-463, [110], pls. I-IX (Tesis doctoral, Universidad Complutense de Madrid).

Maldonado, A., 1985. Evolution of the Mediterranean basins and a detailed reconstruction of the cenozoic paleoceanography. In: R. Margalef ed., Western Mediterranean: 17-59 (Pergamon Press, Oxford, etc.).

Margalef, R., 1983. Limnología: 1-1010 (Ed. Omega, Barcelona).

Mattauer, M. \& J. Henry, 1974. Pyrenees. In: A. M. Spencer ed., Mesozoic-Cenozoic orogenic belts. Data for orogenic studies. Geol. Soc., Spec. Publ., 4: 3-20 (The Geological Society, London).

Matthaeis, E. de, R. Colognola, V. Sbordoni, M. Cobolli Sbordoni \& G. L. Pesce, 1982. Genetic differentiation and variability in cave dwelling and brackish water populations of Mysidae (Crustacea). Z. zool. Syst. Evolutionsforsch., 20: 198-208.

Matthaeis, E. de, G. Allegrucci, A. Caccone, D. Cesaroni, M. Cobolli Sbordoni \& V. Sbordoni, 1983. Genetic differentiation between Penaus kerathurus and P. japonicus (Crustacea, Decapoda). Mar. Ecol. Prog. Ser., 12: 191-197.

Menken, S. B. J. \& S. A. Ulenberg, 1987. Biochemical characters in agricultural entomology. Agric. Zool. Rev., 2: 305-360.

Mickevich, M. F. \& C. Mitter, 1981. Treating polymorphic characters in systematics: a phylogenetic treatment of electrophoretic data. In: V. A. Brooks \& D. R. Brooks eds., Advances in cladistics: Proceedings of the first meeting of the Willi Hennig Society: 4548 (New York Botanical Garden, Bronx).

Mulley, J. C. \& B. D. H. Latter, 1980. Genetic variation and evolutionary relationships within a group of thirteen species of penaeid prawns. Evolution, 34 (5): 904-916.

NeI, M., 1972. Genetic distance between populations. Am. Nat., 106: 283-292.

-, 1975 . Molecular population genetics and evolution: 1.525 (North-Holland, Amsterdam).

Patton, J. C. \& J. C. Avise, 1983. An empirical evaluation of qualitative Hennigian analysis of protein electrophoretic data. J. molec. Evol., 19: 244-254. 
Pinkster, S., 1971. Members of the Gammarus pulexgroup (Crustacea-Amphipoda) from North Africa and Spain, with description of a new species from Morocco. Bull. zoöl. Mus. Univ. Amsterdam, 2 (7): 45-61.

- 1 1983. The value of morphological characters in the taxonomy of Gammarus. Beaufortia, 33 (2): 15-28.

Pinkster, S. \& A. Scholl, 1984. Gammarus orinos n. sp. from the Massif Central (France): its genetic and morphological distinction from Gammarus ibericus Margalef, 1951 (Crustacea, Amphipoda). Bijdr. Dierk., 54 (1): 139-146.

Plaziat, J.-Cl., 1981. Late Cretaceous to Late Eocene palaeogeographic evolution of southwest Europe. Palaeogeography, Palaeoclimatology, Palaeoecology, 36: 263-320.

Poulık, M. D., 1957. Starch gel electrophoresis in a discontinuous system of buffers. Nature, 180: 1477-1479.

Rögl, F. F. F. Steininger, 1983. Vom Zerfall der Tethys zu Mediterran und Paratethys. Die neogene Paläogeographie und Palinspastik des zirkummediterranen Raumes. Annln. naturh. Mus. Wien, 85A: 135-163, 14 maps.

Rogers, J. S., 1972. Measures of genetic similarity and genetic distance. Studies in Genetics, Univ. Texas Publ. 7213: 145-153.

-, 1984 . Deriving phylogenetic trees from allele frequencies. Syst. Zool., 33 (1): 52-63.

-, 1986 . Deriving phylogenetic trees from allele frequencies: a comparison of nine genetic distances. Syst. Zool., 35 (3): 297-310.

Roux, A. L., 1971a. Les Gammares du groupe pulex, III. Essais d'hybridation et examen caryologique. Archs. Zool. exp. gén., 112: 819-868.

- $1971 \mathrm{~b}$. Génétique des populations. Sympatrie, allopatrie et isolement sexuel interspécifique chez les Gammares du groupe pulex. C. r. hebd. Séanc. Acad. Sci., Paris, (D) 273: 408-410.

SARICH, V. M., 1977. Rates, sample sizes, and the neutrality hypothesis for electrophoresis in evolutionary studies. Nature, 265: 24-28.

Sbordoni, V., 1982. Advances in speciation of cave animals. In: C. Barigozzl ed., Mechanisms of speciation: 219-240 (Liss, New York).

Sbordoni, V., A. Caccone, E. de Matthaeis $M$. Cobolli Sbordoni, 1980. Biochemical divergence between cavernicolous and marine Sphaeromidae and the Mediterranean salinity crisis. Experientia, 36: 48-49.

Scheepmaker, M., 1987. Morphological and genetic differentiation of G. stupendus Pinkster, 1983 in the
Massif de la Sainte Baume, France. Bijdr. Dierk., 57 (1): 1-18.

Scholl, A., B. Corzillius \& W. Villwock, 1978. Beitrag zur Verwandtschaftsanalyse altweltlicher Zahnkarpfen der Tribus Arphaniini (Pisces, Cyprinodontidae) mit Hilfe electrophoretischer Untersuchungsmethoden. Z. zool. Syst. Evolutionsforsch., 16: 116-132.

Selander, R. K., 1976. Genic variation in natural populations. In: F. J. Ayala ed., Molecular evolution: 21-45 (Sinauer, Sunderland, Mass.).

Selander, R. K., M. H. Smith, S. Y. Yang, W. E. Johnson \& J. B. Gentry, 1971. Biochemical polymorphisms and systematics in the genus Peromyscus, I. Variation in the old-field mouse. Studies in Genetics, 6. Univ. Texas Publ. 7103 (6): 49-90.

Shaw, C. R. R. Prasad, 1970. Starch gel electrophoresis of enzymes. A compilation of recipes. Biochem. Genet., 4: 297-320.

Siegismund, H. R., V. Simonsen \& S. Kolding, 1985. Genetic studies of Gammarus, I. Genetic differentiation of local populations. Hereditas, 102: 1-13.

Skadsheim, A. H. R. Siegismund, 1986. Genetic relationships among north-western European Gammaridae (Amphipoda). Crustaceana, 51 (2): 163-175.

Sneath, P. H. A. R. R. Sokal, 1973. Numerical taxonomy: i-xv, 1-780 (W. H. Freeman \& Company, San Francisco).

SwOFFord, D. L., 1981. On the utility of the distance Wagner procedure. In: V. A. Brooks \& D. R. Brooks eds., Advances in cladistics: Proceedings of the first meeting of the Willi Hennig Society: 25-43 (New York Botanical Garden, Bronx).

Swofford, D. L. \& S. H. Berlocher, 1987. Inferring evolutionary trees from gene frequency data under the principle of maximum parsimony. Syst. Zool., 26 (3): 293-325.

Swofford, D. L. R. B. Selander, 1981. Biosys-1. A computer program for the analysis of allelic variation in genetics. (Dept. of Genetics and Development, Univ. of Illinois at Urbana-Champaign, Urbana, Illinois.)

ThORPE, J., 1982. The molecular clock hypothesis: biochemical evolution, genetic differentiation and systematics. Annual Rev. Ecol. Syst., 13: 139-168.

VANDENBERG, J., 1979. Reconstruction of the western Mediterranean area for the Mesozoic and Tertiary timespan. Geol. Mijnbouw, 58 (2): 153-160.

Wautier, J. A. L. Roux, 1959. Note sur les Gammares du groupe pulex dans la région Lyonnaise. Bull. mens. Soc. linn. Lyon, 28 (3): 76-83. 